\title{
A HOPF GLOBAL BIFURCATION THEOREM FOR RETARDED FUNCTIONAL DIFFERENTIAL EQUATIONS
}

BY

ROGER D. NUSSBAUM ${ }^{1}$

\begin{abstract}
We prove a result concerning the global nature of the set of periodic solutions of certain retarded functional differential equations. Our main theorem is an analogue, for retarded F.D.E.'s, of a result by $J$. Alexander and $\mathrm{J}$. Yorke for ordinary differential equations.
\end{abstract}

Introduction. In the past ten or fifteen years there has been considerable interest in the global nature of the set of periodic solutions of certain parametrized families of F.D.E.'s. These equations arise in a variety of applications, for example, mathematical biology [19]. References at the end of this paper give some guidance to the relevant literature.

For those equations to which it is applicable, the global bifurcation theorem in [21] appears to provide the sharpest global information. However, there are simple-looking F.D.E.'s for which the results of [21] are not easily applicable. We mention one example; consider the equation

$$
x^{\prime}(t)=[-\alpha x(t-1)-c \alpha x(t-\gamma)]\left[1-x^{2}(t)\right],
$$

where $c$ and $\gamma$ are positive constants, $1 \leqslant \gamma \leqslant 2$ and $\alpha>0$. Let $\alpha_{0}$ denote the smallest positive $\alpha$ such that the equation

$$
z=-\alpha e^{-z}-c \alpha e^{-\gamma z}
$$

has a pair of pure imaginary solutions. For a variety of reasons, it is reasonable to conjecture that for every $\alpha>\alpha_{0}$, (1) has a "slowly oscillating" (a term we leave undefined) nonconstant periodic solution. Despite remarks made in [15] for the case $\gamma=2$, this modest conjecture has still not been proved in general. The cases $c=0$ and $c=1$ (for $\gamma=2$ ) treated in [15] are atypical.

Thus it seems reasonable to try to obtain a global bifurcation theorem for periodic solutions which would perhaps provide less detailed information than the one in [21] but which would be more broadly applicable. J. Alexander and $\mathrm{J}$. Yorke have established a generalization of the classical Hopf bifurcation theorem [1], and J. Ize [12], [13] has given a considerable

Received by the editors June 28, 1976.

AMS (MOS) subject classifications (1970). Primary 34K15; Secondary 47H15.

${ }^{1}$ Partially supported by a National Science Foundation Grant.

- American Mathematical Society 1978 
simplification of the original proof. We shall prove here an analogue of the Yorke-Alexander theorem for retarded F.D.E.'s. The proof follows the general outlines of Ize's simplification, but the infinite dimensional nature of the problem and, more importantly, the lack of compactness of certain maps introduce considerable difficulties; and it is the treatment of these difficulties we shall emphasize. The proof we give here can be abstracted to certain evolution equations in Banach spaces, though we do not pursue this. We should remark that if the operation of translation along trajectories (for the evolution equation) is compact, the use of finite dimensional projections given here can be avoided and the proof considerably simplified. The techniques we give can also be used to study the global nature of nonconstant periodic solutions of integral equations like those in [4]. Although our primary interest in the theorem here is its application to specific equations, we defer these applications to [25] because of considerations of length. We hope to show in [25] how a variety of techniques (including Theorem 4 below) can be used to study periodic solutions of, for example, equation (1).

After this paper was written we received a preprint of a paper by Chow and Mallet-Paret in which they outline a proof of a result like Theorem 4 below (for the case $\operatorname{Mult}(i \beta)=\{i \beta\}$ in our later notation). The proof involves approximation of retarded F.D.E.'s by Kupka-Smale systems (as in [18]) and generalizations of Fuller's index [6] to retarded FDE's; presumably such extensions would also be necessary in applying their ideas to other kinds of equations. Chow and Mallet-Paret also give an interesting application to (1) (but only for $\gamma$ an integer) in order to obtain "rapidly oscillating" periodic solutions. However, the existence of slowly oscillating periodic solutions for $\alpha>\alpha_{0}$ does not follow, and it is the slowly oscillating periodic solutions which have been studied numerically and which are of greater interest.

An outline of this paper may be in order. In the first section we prove that the operator of translation along trajectories for retarded F.D.E.'s is strongly approximation proper (strongly $A$-proper) with respect to a natural set of projections $\left\{P_{m}\right\}$, although the operator is not, in general, compact. This observation provides a means of passing from finite dimensional to infinite dimensional results and is extensively used. The second section reviews the linear theory of retarded F.D.E.'s and derives some simple consequences of known results. The third section shows, in Theorem 3, that a certain element of the first homotopy group of $G L^{+}\left(R^{p}\right)$ is nonzero for large $p$, where $G L^{+}\left(R^{p}\right)$ denotes a connected component of the general linear group on $R^{p}$. The main result of the paper is Theorem 4 of $\S 4$, which is an exact analogue of the Yorke-Alexander result.

1. A class of strongly $A$-proper mappings. In this section we shall prove the $A$-properness (see [26]) of a new class of mappings. For technical reasons, this 
result will be crucial for the remainder of the paper.

First we need some notation. Let $\gamma$ be a fixed positive constant and define $X$ to be the Banach space of continuous maps $x:[-\gamma, 0] \rightarrow K^{n}$, where $K$ denotes either the reals or the complexes. The norm is the usual sup norm. For each $m \geqslant 1$ let $t_{0}=-\gamma<t_{1}<t_{2}<\cdots<t_{m}=0$ be a partition of $[-\gamma, 0]$ into $m$ intervals $\Delta_{j}=\left[t_{j-1}, t_{j}\right]$ of equal length and define a finite dimensional linear projection $P_{m}: X \rightarrow X$ by $P_{m} x=y$, where

$$
y(t)=\left(\frac{t_{j}-t}{t_{j}-t_{j-1}}\right) x\left(t_{j-1}\right)+\left(\frac{t-t_{j-1}}{t_{j}-t_{j-1}}\right) x\left(t_{j}\right)
$$

for $t \in \Delta_{j}$. It is easy to check that $\left\|P_{m}\right\|=1$ and that $\lim _{m \rightarrow \infty} P_{m} x=x$ for each $x \in X$. We shall adhere to the above use of $X, \Delta_{j}, t_{j}$ and $P_{m}$ throughout this section.

Next suppose that $Z$ is a Banach space and that $\left\{Q_{m}: m \geqslant 1\right\}$ is a sequence of continuous linear projections with the property that $\lim _{m \rightarrow \infty} Q_{m} z=z$ for every $z \in Z$. Let $B$ be a compact metric space, $A$ a closed subset of $Z \times B$ and $\Phi: Z \rightarrow Z$ a continuous map. Define $\Pi: Z \times B \rightarrow Z$ by $\Pi(z, b)=z$.

Definition 1 . The map $\Pi-\Phi$ is "strongly $A$-proper with respect to $\left\{Q_{m}\right\}$ " if for every subsequence $\left\{m_{i}\right\}$ of the integers and every bounded sequence $\left(a_{m_{i}}, b_{m_{i}}\right) \in A$ such that $a_{m_{i}}-Q_{m_{i}} \Phi\left(a_{m_{i}}, b_{m_{i}}\right)$ is convergent, there exists a further subsequence $\left(a_{m_{i j}}, b_{m_{i j}}\right)$ which is convergent.

The above definition strengthens the usual notion of $A$-properness in that it is not assumed that $a_{m_{i}} \in Q_{m_{i}}(Z)$.

We also need a notion of restricted equicontinuity.

Definition. If $S \subset X$ is a family of functions and $J \subset[-\gamma, 0]$ is a closed subinterval, " $S$ is equicontinuous on $J$ " if the restriction of elements of $S$ to $J$ gives an equicontinuous family on $J$.

TheOREM 1. Let $A$ be a closed subset of $X \times B, B$ a compact metric space, $\Phi: A \rightarrow X$ a continuous map and $\Pi: X \times B \rightarrow X$ the standard projection. Assume that there exists $\varepsilon>0$ such that whenever $A_{1} \subset A$ is closed, bounded and $\Pi\left(A_{1}\right)$ is equicontinuous on an interval $\left[-\rho_{1}, 0\right], 0 \leqslant \rho_{1} \leqslant \gamma$, then $A_{2}=$ $\left\{\Phi(a, b):(a, b) \in A_{1} \times B\right\}$ is closed, bounded and equicontinuous on $\left[-\rho_{2}, 0\right]$, where $\rho_{2}=\min \left(\rho_{1}+\varepsilon, \gamma\right)$. Then it follows that $\Pi-\Phi$ is strongly $A$-proper with respect to $\left\{P_{m}\right\}$.

Proof. Let $\left(a_{m_{i}}, b_{m_{i}}\right) \in A$ be a bounded sequence such that

$$
a_{m_{i}}-P_{m_{i}} \Phi\left(a_{m_{i}}, b_{m_{i}}\right)=z_{m_{i}} \rightarrow z \text {. }
$$

By relabelling the projections and using the compactness of $B$, we can write $m_{i}=m$ and assume $b_{m} \rightarrow b$. According to the Ascoli-Arzela theorem, it suffices to show $\left\{a_{m}: m \geqslant 1\right\}$ is equicontinuous on $[-\gamma, 0]$. The latter will 
follow by a bootstrap argument if we can prove that $\left\{a_{m}\right\}$ equicontinuous on $[-\rho, 0], 0 \leqslant \rho \leqslant \gamma$, implies that $\left\{a_{m}\right\}$ is equicontinuous on $\left[-\rho_{1}, 0\right]$, where $\rho_{1}=\min (\gamma, \rho+\varepsilon / 2)$.

Thus suppose we have shown that $\left\{a_{m}: m \geqslant 1\right\}$ is equicontinuous on $[-\rho, 0]$. Since $\left\{z_{m}\right\}$ is convergent and, hence, equicontinuous on $[-\gamma, 0],(4)$ shows that it suffices to prove $P_{m} \Phi\left(a_{m}, b_{m}\right)$ is equicontinuous on $\left[-\rho_{1}, 0\right]$. By assumption, $w_{m}=\Phi\left(a_{m}, b_{m}\right)$ is equicontinuous on $\left[-\rho_{2}, 0\right]$, where $\rho_{2}=$ $\min (\gamma, \rho+\varepsilon)$. If $\rho_{2}=\gamma$, we claim that $\left\{P_{m} w_{m}: m \geqslant 1\right\}$ is equicontinuous on $[-\gamma, 0]$. To see this, given $\eta>0$ select $\delta>0$ such that $|t-s|<\delta$ implies that $\left|w_{m}(t)-w_{m}(s)\right|<\eta / 3$ for all $m \geqslant 1$. An easy argument using the definition of $P_{m}$ shows that if $m \geqslant N$, where $\gamma / N<\delta$, and $|t-s|<\delta$, then $\left|P_{m} w_{m}(t)-P_{m} w_{m}(s)\right|<\eta$. This shows that $\left\{P_{m} w_{m}: m \geqslant N\right\}$ is equicontinuous on $[-\gamma, 0]$, and, consequently, $\left\{P_{m} w_{m}: m \geqslant 1\right\}$ is equicontinuous on $[-\gamma, 0]$. In the case that $\rho_{2}=\rho+\varepsilon<\gamma$, if one takes $\eta$ and $\delta$ as above and $N$ such that $\gamma / N<\min (\delta, \varepsilon / 2)$, then the same sort of argument used above works to show $\left\{P_{m} w_{m}: m \geqslant 1\right\}$ is equicontinuous on $\left[-\rho_{1}, 0\right]$.

Our interest in Theorem 1 stems from its applicability to the operator of translation along trajectories for retarded functional differential equations (F.D.E.'s). Specifically, let $X$ be as usual, with scalar field the reals, and let $\Lambda$ denote an open interval of real numbers. We shall henceforth denote by $f$ : $X \times \Lambda \rightarrow R^{n}$ a map such that:

H1. $f: X \times \Lambda \rightarrow R^{n}$ is continuous and takes bounded sets in $X \times \Lambda$ to bounded sets in $\mathbf{R}^{n}$.

Following the notation in [10], we are interested in nonconstant periodic solutions of

$$
x^{\prime}(t)=f\left(x_{t}, \lambda\right) .
$$

For each $\phi \in X$, we can consider the initial value problem

$$
x^{\prime}(t)=f\left(x_{t}, \lambda\right) \text { for } t \geqslant 0, \quad x \mid[-\gamma, 0]=\phi .
$$

We must assume that:

H2. For each $\phi \in X$ and $\lambda \in \Lambda$ equations (6) have a unique solution $x(t)=x(t ; \phi, \lambda)$ defined and continuous on $[-\gamma, \delta)$ for some positive $\delta$ and $C^{1}$ on $[0, \delta)$.

A standard argument shows that $x(t ; \phi, \lambda)$ can be extended to some maximal, half-open interval of definition $[-\gamma, t(\phi, \lambda))$. Furthermore, if $G=$ $\{(\phi, \lambda, t) \in X \times \Lambda \times[0, \infty): x(t ; \phi, \lambda)$ is defined $\}$ arguments like those for O.D.E.'s show that $G$ is an open subset of $X \times \Lambda \times[0, \infty)$ and the map $(\phi, \lambda, t) \rightarrow x(t ; \phi, \lambda)$ is continuous; see [10] for details. We shall reserve the letter $G$ to denote the above set.

Unfortunately, an example of $\mathrm{K}$. Hannsgen (see [10, p. 39]) shows that $x(t ; \phi, \lambda)$ may not be bounded on closed, bounded subsets of $G$; since we 
shall need this boundedness we assume:

H3. If $A$ is any closed, bounded subset of $G$, the function $x(t ; \phi, \lambda)$ is bounded on $A$.

Assuming that $\mathrm{H} 1, \mathrm{H} 2$ and $\mathrm{H} 3$ hold, define a map

(7)

$$
F: G \rightarrow X
$$

by $F(\phi, \lambda, t)=x_{t}$, where $x(t)=x(t ; \phi, \lambda)$ is the unique solution of (6). We shall always use $F$ to denote this map. Let $\Pi: X \times \Lambda \times[0, \infty) \rightarrow X$ be projection onto $X$.

TheOREM 2. Assume that $\mathrm{H} 1, \mathrm{H} 2$ and $\mathrm{H} 3$ hold and let $A$ be a closed, bounded subset of $G$ such that $\inf \{t:(\phi, \lambda, t) \in A$ for some $\phi$ and $\lambda\}=\varepsilon>0$. Then the map $\Pi-F \mid A$ is strongly $A$-proper with respect to $\left\{P_{m}\right\}$.

Proof. It suffices to show that $\Phi=F$ satisfies the hypothesis of Theorem 1. Let $A_{1}$ be a subset of $A$ such that $\Pi\left(A_{1}\right)$ is equicontinuous on [ $\left.-\rho_{1}, 0\right]$. If $(\phi, \lambda, t) \in A_{1}$, consider $x(s ; \phi, \lambda)$ for $0 \leqslant s \leqslant t$, and note that by $\mathrm{H} 3$ and the boundedness of $A_{1}$ there is a constant $M$ (independent of $\left.(\phi, \lambda, t) \in A_{1}\right)$ such that

$$
|x(s ; \phi, \lambda)| \leqslant M
$$

for $-\gamma<s \leqslant t$. The boundedness of $f$ now implies that there is a constant $M_{1}$ such that

$$
\left|x^{\prime}(s ; \phi, \lambda)\right| \leqslant M_{1}
$$

for $0 \leqslant s \leqslant t$. Since $t \geqslant \varepsilon$ for every $(\phi, \lambda, t) \in A$, it follows from (9) and the assumption that $\Pi\left(A_{1}\right)$ is equicontinuous on $\left[-\rho_{1}, 0\right]$ that $\{F(\phi, \lambda, t):(\phi, \lambda, t)$ $\left.\in A_{1}\right\}$ is equicontinuous on $\left[-\rho_{2}, 0\right], \rho_{2}=\min \left(\rho_{1}+\varepsilon, \gamma\right)$.

2. Linear theory of retarded functional differential eqations. In this section we shall recall for the reader's convenience some basic facts about linear retarded F.D.E.'s (further details appear in [10]) and derive some simple consequences. As usual, let $X$ ( $\tilde{X}$ respectively) denote the continuous functions $[-\gamma, 0]$ to $\mathbf{R}^{n}$ (to $\mathbf{C}^{n}$ respectively). Suppose that $L: \tilde{X} \rightarrow \mathbf{C}^{n}$ is a bounded linear map and consider

$$
x^{\prime}(t)=L\left(x_{t}\right) \text { for } t \geqslant 0, \quad x \mid[-\gamma, 0]=\phi \in \tilde{X} .
$$

For each $\phi \in \tilde{X},(10)$ has a unique solution $x(t ; \phi)$ defined for $t \geqslant-\gamma$. The map $\phi \rightarrow x_{t}=T(t)(\phi)$ defines a bounded linear operator $T(t)$ and $\{T(t)$ : $t \geqslant 0\}$ gives a strongly continuous, linear semigroup on $\tilde{X}$. The infinitesimal generator $A$ of the semigroup $T(t)$ is given by

$$
\begin{gathered}
(A \phi)(s)=\phi^{\prime}(s) \text { for }-\gamma \leqslant s \leqslant 0 ; \\
D(A)=\text { domain of } A=C^{1} \text { functions } \phi \in \tilde{X} \\
\text { such that } \phi^{\prime}(0)=L(\phi) .
\end{gathered}
$$


For each complex number $z$ define a linear map $\Delta(z): \mathbf{C}^{n} \rightarrow \mathbf{C}^{n}$ by the formula

$$
\Delta(z)(b)=z b-L\left(e^{z s} b\right),
$$

where $b$ denotes a vector in $\mathbf{C}^{n}$ and $e^{z s} b$ denotes the map $s \in[-\gamma, 0] \rightarrow e^{z s} b$. One can check directly that the map $z \rightarrow \Delta(z)$ is complex analytic (and not identically zero), so the map $z \rightarrow(\operatorname{det}(\Delta(z)))^{-1}$ is meromorphic (det denotes determinant). We shall need the fundamental facts that $\sigma(A)=$ the point spectrum of $A$ and that $\sigma(A)=\{z \in \mathbf{C}$ : $\operatorname{det} \Delta(z)=0\}$. Furthermore, recall that since $T(t)^{m}$ is a compact linear operator for $m t \geqslant \gamma$, it follows that $\sigma(T(t))=$ the point spectrum of $T(t)$ and that (compare [11, p. 467] and [10, p. 112]) $\sigma(T(t))-\{0\}=\{\exp (t z): z \in \sigma(A)\}$.

We shall also need an explicit formula for $(z-A)^{-1}(\psi)=\phi$ (assuming $\operatorname{det} \Delta(z) \neq 0)$. One can check that

$$
\begin{aligned}
& \phi(t)=e^{z t} b+\int_{t}^{0} e^{z(t-s)} \psi(s) d s \\
& \text { where } b=\Delta(z)^{-1}\left[\psi(0)+L\left(\int_{t}^{0} e^{z(t-s)} \psi(s) d s\right)\right] .
\end{aligned}
$$

Next suppose that $\Lambda$ is an open interval of reals, and that for each $\lambda \in \Lambda$, $L_{\lambda}: \tilde{X} \rightarrow C^{n}$ is a continuous map and $\lambda \rightarrow L_{\lambda}$ is continuous in the uniform operator topology. In the obvious notation we can consider the strongly continuous linear semigroup $T_{\lambda}(t)(t \geqslant 0)$ generated by solving

$$
x^{\prime}(t)=L_{\lambda}\left(x_{t}\right), \quad t \geqslant 0 ; \quad x \mid[-\gamma, 0]=\phi,
$$

the infinitesimal general $A_{\lambda}$ of $T_{\lambda}(t)$ and $\Delta_{\lambda}(z)$ defined by a formula like (12) with $L_{\lambda}$ substituted for $L$. We shall maintain this notation for the rest of the paper.

It follows directly from (12) that if $\lambda \rightarrow L_{\lambda}$ is continuous, $\Delta_{\lambda}(z) \rightarrow \Delta_{\lambda_{0}}(z)$ as $\lambda \rightarrow \lambda_{0}$ uniformly for $z$ in a compact set. Since $\sigma\left(A_{\lambda}\right)=\left\{z \in \mathbf{C}\right.$ : $\operatorname{det} \Delta_{\lambda}(z)=$ $0\}$, we see that if $\Gamma$ is any compact subset of the resolvent set $\rho\left(A_{\lambda_{0}}\right)$ of $A_{\lambda_{0}}$, then for $\left|\lambda-\lambda_{0}\right|<\varepsilon, \Gamma$ is in the resolvent set of $A_{\lambda}$. Furthermore, (13) implies that

$$
\left\|\left(z-A_{\lambda}\right)^{-1}-\left(z-A_{\lambda_{0}}\right)^{-1}\right\| \rightarrow 0
$$

as $\lambda \rightarrow \lambda_{0}$, uniformly in $z \in \Gamma$.

We shall also need some elementary results from the functional calculus for linear operators. Let $G$ be a bounded open set whose boundary consists of a finite number of simple, closed Jordan curves which lie in the resolvent set of a closed, densely defined linear operator $B$ on a complex Banach space $Z$. One can consider a bounded linear operator 


$$
P=\frac{1}{2 \Pi i} \int_{\Gamma}(z-B)^{-1} d z .
$$

The operator $P$ is a projection whose range lies in the domain of $B$. If $\Gamma$ contains only one point $z_{0}$ of $\sigma(B)$, and the Laurent expansion of $(z-B)^{-1}$ at $z_{0}$ has only finitely many terms with negative indices so that

$$
(z-B)^{-1}=\sum_{j=-k}^{\infty} C_{j}\left(z-z_{0}\right)^{j}
$$

with $C_{j}$ bounded and $C_{-k} \neq 0$, then $R(P)=$ range of $P$ is finite dimensional and $R(P)$ is the null space of $\left(z_{0}-B\right)^{k}=N\left(\left(z_{0}-B\right)^{k}\right)$, which is the same as the null space of $\left(z_{0}-B\right)^{j}$ for $j \geqslant k$ (see [17, p. 29] and [11, Chapter 5]). Let $\sigma_{1}$ denote the part of $\sigma(B)$ which lies inside $\Gamma, \sigma_{2}=\sigma(B)-\sigma_{1}, Z_{1}=$ $R(P)$ and $Z_{2}=R(Q)$, where $Q=I-P$; also denote by $B_{1}$ the restriction of $B$ to $Z_{1}$ and by $B_{2}$ the restriction of $B$ to $Z_{2}$. Then it follows (see Theorem 6.17, p. 178 in [17]) that $\sigma_{j}$ is the spectrum of $B_{j}$.

In our case we take $A_{\lambda}$ to be $B$ and $\Gamma$ to be a union of curves as above in the resolvent of $A_{\lambda}$, and we define projections $P_{\lambda}$ and $Q_{\lambda}$ by

$$
P_{\lambda}=\frac{1}{2 \pi i} \int_{\Gamma}\left(z-A_{\lambda}\right)^{-1} d z, \quad Q_{\lambda}=I-P_{\lambda},
$$

and set $\tilde{X}_{\lambda}=P_{\lambda}(\tilde{X}), \tilde{Y}_{\lambda}=Q_{\lambda}(\tilde{X}), X_{\lambda}=X \cap \tilde{X}_{\lambda}$ and $Y_{\lambda}=X \cap \tilde{Y}_{\lambda}$. Again, we shall maintain this notation from now on. Let $\sigma_{1}$ denote the finite number of points in $\sigma\left(A_{1}\right)$ which lie inside $\Gamma$ and $\sigma_{2}=\sigma\left(A_{\lambda}\right)-\sigma_{1}$.

REMARK 1. In general, $\Gamma$ must be chosen to vary with $\lambda$, but the previous remarks show that if $\Gamma$ is permissible for $\lambda_{0}$, it is also permissible for $\left|\lambda-\lambda_{0}\right|\left\langle\varepsilon, \varepsilon>0\right.$. For these $\lambda$, (15) shows that $\lambda \rightarrow P_{\lambda}$ is continuous (assuming $\lambda \rightarrow L_{\lambda}$ is continuous).

Our next proposition can be found, for the most part, in [10, pp. 94-115]; we sketch a proof for completeness.

Proposition 1. Let notation be as above. Then it follows that $\tilde{X}=\tilde{X}_{\lambda} \oplus \tilde{Y}_{\lambda}$, $\tilde{X}_{\lambda}$ is finite dimensional, $T_{\lambda}(t)\left(\tilde{X}_{\lambda}\right) \subset \tilde{X}_{\lambda}$ and $T_{\lambda}(t)\left(\tilde{Y}_{\lambda}\right) \subset \tilde{Y}_{\lambda}$ for $t \geqslant 0$. Furthermore, for $t>0$ the spectrum of $T_{\lambda}(t) \mid \tilde{X}_{\lambda}$ equals $\left\{\exp (t s): s \in \sigma_{1}\right\}$, and the spectrum of $T_{\lambda}(t) \mid \tilde{Y}_{\lambda}$ equals $\left\{\exp (t s): s \in \sigma_{2}\right\}$.

PROOF. The general theory of linear semigroups implies that $T_{\lambda}(t)$ and $A_{\lambda}$ commute on the domain of $A_{\lambda}$; from this one obtains that $T_{\lambda}(t)$ and $\left(z-A_{\lambda}\right)^{-1}$ commute and, hence, $T_{\lambda}(t)$ and $P_{\lambda}$ commute. Thus $T_{\lambda}(t)$ maps $\tilde{X}_{\lambda}$ and $\tilde{Y}_{\lambda}$ into themselves. The general theory of the functional calculus for linear operators implies that $\tilde{X}=\tilde{X}_{\lambda} \otimes \tilde{Y}_{\lambda}, P_{\lambda}$ is a projection and $X_{\lambda}$ is in the domain of $P_{\lambda}$. Since $G_{j}$, the interior of $\Gamma$, is assumed bounded and $\sigma\left(A_{\lambda}\right)$ is discrete, $\sigma_{1}$ consists of a finite number of points. Thus to show $X_{\lambda}$ is finite dimensional, it suffices to show $\left(z-A_{\lambda}\right)^{-1}$ is meromorphic at any point $z_{0}$ 
such that det $\Delta\left(z_{0}\right)=0$; in fact it follows from (13) that if $z_{0}$ is a zero of det $\Delta(z)$ of order $k$, then $\left(z-A_{\lambda}\right)^{-1}$ has a pole of order $k$ at $z_{0}$.

It is clear that $T_{\lambda}(t) \mid \tilde{X}_{\lambda}$ and $T_{\lambda}(t) \mid \tilde{Y}_{\lambda}$ are strongly continuous semigroups with generators $A_{\lambda} \mid \tilde{X}_{\lambda}$ and $A_{\lambda} \mid \tilde{Y}_{\lambda}$. Theorem $6.17[17, \mathrm{p}$. 178] implies that $\sigma\left(A_{\lambda} \mid \tilde{X}_{\lambda}\right)=\sigma_{1}$ and $\sigma\left(A_{\lambda} \mid \tilde{Y}_{\lambda}\right)=\sigma_{2}$; it follows from Theorem 16.7.2 [11, p. 467] that for $t>0$, the spectrum of $T_{\lambda}(t) \mid \tilde{X}_{\lambda}$ is $\left\{\exp (t s): s \in \sigma_{1}\right\}$ plus possibly 0 , and similarly for $T_{\lambda}(t) \mid \tilde{Y}_{\lambda}$. However, a strongly continuous semigroup on a finite dimensional space is necessarily continuous in the uniform operator topology. Thus $T_{\lambda}(t) \mid \tilde{X}_{\lambda}$ is invertible for $t$ small, and, hence, $T_{\lambda}(t) \mid \tilde{X}_{\lambda}$ is invertible for all $t \geqslant 0$ and 0 is not in its spectrum. On the other hand, $\tilde{Y}_{\lambda}$ is infinite dimensional and, for each $t>0, T_{\lambda}(t)^{N}$ is compact for some $N$. It follows that 0 cannot be in the resolvent of $T_{\lambda}(t) \mid \tilde{Y}_{\lambda}$ (if it were, the unit ball in $\tilde{Y}_{\lambda}$ would be compact).

If $L: \tilde{X} \rightarrow C^{n}$ is a bounded linear function, let $A$ and $T(t)$ be defined as at the beginning of this section. Suppose that $L(X) \subset \mathbf{R}^{n}$ (where $X=$ $\left.C\left([-\gamma, 0] ; \mathbf{R}^{n}\right)\right)$; then it is easy to check that $A$ and $T(t)$ map $X$ into $X$. If $z_{j}$ and $\bar{z}_{j}, 1 \leqslant j \leqslant k$, are $k$ pairs of conjugate complex numbers (we allow $z_{j}=\bar{z}_{j}$ ) such that $z_{j}$ and $\bar{z}_{j}$ are elements of $\sigma(A)$, let $\Gamma_{j}$ and $\bar{\Gamma}_{j}$ be simple, closed curves containing, respectively, $z_{j}$ and $\bar{z}_{j}$ and no other points of $\sigma(A)$. Let $\Gamma$ denote the union of these curves and define

$$
\begin{aligned}
P & =\frac{1}{2 \pi i} \int_{\Gamma}(z-A)^{-1} d z \\
& =\frac{1}{2 \pi i} \sum_{j=1}^{k} \int_{\Gamma_{j}}(z-A)^{-1} d z+\int_{\bar{\Gamma}_{j}}(z-A)^{-1} d z .
\end{aligned}
$$

Proposition 2. Let assumptions be as in the preceding paragraph. Then it follows that $P(X) \subset X$.

Proof. It suffices by (18) to prove the proposition in the case of two points $z_{j}$ and $\bar{z}_{j}$ (possibly equal). We assume $z_{j} \neq \bar{z}_{j}$, since the proof in the case $z_{i}=\bar{z}_{j}$ is essentially the same. Since $(z-A)^{-1}$ is analytic on the interior of $\Gamma_{j}$ $\left(\bar{\Gamma}_{j}\right)$ except at $z_{j}\left(\bar{z}_{j}\right)$, we can assume that $\Gamma_{j}$ and $\bar{\Gamma}_{j}$ are circles $C$ and $\bar{C}$, respectively, with radius $r$ and centers at $z_{j}$ and $\bar{z}_{j}$, respectively. If $\phi \in X$, a simple calculation gives that

$$
\begin{aligned}
P \phi=\frac{r}{2 \pi} \int_{\theta=0}^{2 \pi}\left[\left(z_{j}\right.\right. & \left.+r e^{i \theta}-A\right)^{-1}\left(e^{i \theta} \phi\right) \\
& \left.+\left(\bar{z}_{j}+r e^{-i \theta}-A\right)^{-1}\left(e^{-i \theta} \phi\right)\right] d \theta .
\end{aligned}
$$

(19) shows that to prove the proposition it suffices to prove that

$$
(z-A)^{-1}\left(e^{i \theta} \phi\right)+(\bar{z}-A)^{-1}\left(e^{-i \theta} \phi\right)
$$


is an $\mathbf{R}^{n}$-valued function whenever $\phi$ is. However, since $A(X) \subset X$ it is easy to check that if $x \in \tilde{X}$ and $y=(z-A)^{-1}(x)$, then $\bar{y}=(\bar{z}-A)^{-1}(\bar{x})$, which shows the expression in (20) is $\mathbf{R}^{n}$-valued.

If $L: \tilde{X} \rightarrow C^{n}$ is a bounded linear map and $z_{0} \in \sigma(A)$, so det $\Delta\left(z_{0}\right)=0$, we shall need the idea of the algebraic multiplicity of $z_{0}$.

Definition 2. Let $\Gamma$ be a circle containing $z_{0}$ and no other points of $\sigma(A)$ and define a projection $P: \tilde{X} \rightarrow \tilde{X}$ by $P=(2 \pi i)^{-1} \int_{\Gamma}(z-A)^{-1} d z$. Then the algebraic multiplicity of $z_{0}$ is the dimension of the range of $P$.

Several comments are in order. If det $\Delta(z)=\left(z-z_{0}\right)^{k} g(z)$, where $g\left(z_{0}\right) \neq$ 0 , then our previous comments show that the algebraic multiplicity of $z_{0}$ is the dimension of the null space of $\left(z_{0}-A\right)^{k}$. If $L_{\lambda}: \tilde{X} \rightarrow C^{n}$ for $\lambda \in \Lambda, \lambda \rightarrow L_{\lambda}$ is continuous, $z_{0}$ is a zero of det $\Delta_{\lambda_{0}}(z)$ of multiplicity $k$, and $\Gamma$ is a circle about $z_{0}$, then Rouche's theorem implies that for $\left|\lambda-\lambda_{0}\right|<\delta(\delta>0)$, det $\Delta_{\lambda}(z)$ will have a total of $k$ zeros inside $\Gamma$ (counting algebraic multiplicity).

Furthermore, if $\delta$ is so small that $P_{\lambda}=(2 \pi i)^{-1} \int_{\Gamma}(z-A)^{-1} d z$ is defined and $\left\|P_{\lambda}-P_{\lambda_{0}}\right\|<1$ for $\left|\lambda-\lambda_{0}\right|<\delta$, then the dimension of the range of $P_{\lambda}$ will be the same as the dimension of the range of $P_{\lambda_{0}}$, in fact if $P_{1}$ and $P_{2}$ are projections on a general Banach space and $\left\|P_{1}-P_{2}\right\|<1$, then the range of $P_{1}$ and $P_{2}$ have the same dimension.

Finally, suppose $L: \tilde{X} \rightarrow C^{n}$ and det $\Delta\left(z_{j}\right)=0$ for $1 \leqslant j \leqslant k$. Let $\Gamma$ be a circle about $z_{j}$ which contains no other points of $\sigma(A)$, let $\Gamma=\Gamma_{j}$ and define

$$
\begin{aligned}
P & =\frac{1}{2 \pi i} \int_{\Gamma}(z-A)^{-1} d z \\
& =\sum_{j=1}^{k} \frac{1}{2 \pi i} \int_{\Gamma_{j}}(z-A)^{-1} d z=\sum_{j=1}^{k} P_{j \cdot} .
\end{aligned}
$$

Since $P_{l} P_{m}=P_{m}$ for $l=m$ and 0 otherwise, the dimension of the range of $P$ is $\sum_{j=1}^{k} n_{j}$, where $n_{j}$ is the algebraic multiplcity of $z_{j}$. If $V$ is the range of $P$, $A \mid V=B$ is a bounded linear operator, and it makes sense to talk about the characteristic polynomial $\operatorname{det}(z I-B)=p(z)$ of $B$. General facts about the functional calculus imply that $z_{j}, j=1, \ldots, k$, are the roots of $p(z)$ and that $n_{j}$ is the algebraic multiplicity of root $z_{j}$.

3. A nontrivial element of $\Pi_{1}\left(G L_{m}^{+}\right)$. We return now to the study of (6) in \$1. In addition to $\mathrm{H} 1-\mathrm{H} 3$ we suppose

H4. The function $f(\phi, \lambda)$ satisfies

$$
f(\phi, \lambda)=L_{\lambda}(\phi)+R(\phi, \lambda)
$$

where $L_{\lambda}: X \rightarrow X$ is a continuous linear map and $R(\phi, \lambda)=o(\|\phi\|)$ uniformly on compact $\lambda$-intervals. The map $\lambda \rightarrow L_{\lambda}$ is continuous in the uniform operator topology.

REMARK 2. The assumption that $\lambda \rightarrow L_{\lambda}$ is continuous in the uniform 
operator topology is restrictive. For example, if $\left(L_{\lambda} \phi\right)=\phi(g(\lambda))$, where $g(\lambda)$ is a continuous function of $\lambda$ such that $-\gamma \leqslant g(\lambda) \leqslant 0$, the map $\lambda \rightarrow L_{\lambda}$ is continuous in the strong operator topology, but not the uniform operator topology. Actually, the results of this paper carry over if $\lambda \rightarrow L_{\lambda}$ is only continuous in the strong operator topology, but for simplicity we restrict ourselves to $\mathrm{H} 4$.

Now assume that $\mathrm{H} 1-\mathrm{H} 4$ hold, let $F(\phi, \lambda, t)$ be defined by (7) and $T_{\lambda}(t)$ defined as in $\$ 2$.

Lemma 1. Assume that $\mathrm{H} 1-\mathrm{H} 4$ hold and let $M$ be a compact subset of $\Lambda \times \mathbf{R}^{+}$. Then given $\varepsilon>0$, there exists $\delta=\delta(\varepsilon, M)>0$ such that

$$
\left\|F(\phi, \lambda, t)-T_{\lambda}(t)(\phi)\right\| \leqslant \varepsilon\|\phi\|
$$

whenever $(\lambda, t) \in M$ and $\|\phi\| \leqslant \delta$.

Proof. This is an exercise in the variation of constants formula for F.D.E.'s. First, by the continuity of $F$, we can assume $\delta_{1}$ chosen such that $F(\phi, \lambda, t)$ is defined for $\|\phi\| \leqslant \delta_{1}$ and $(\lambda, t) \in M$. By the variation of constants formula we have

$$
F(\phi, \lambda, t)=T_{\lambda}(t) \phi+\int_{0}^{t} T_{\lambda}(t-s) R\left(x_{s}, \lambda\right) d s,
$$

where $x(s ; \phi, \lambda)$ is the solution of (6). It is known (and not hard to prove) that

$$
\left\|T_{\lambda}(u)\right\| \leqslant \exp \left(\left\|L_{\lambda}\right\| u\right) .
$$

It follows that there is a constant $C$ such that

$$
\left\|T_{\lambda}(t-s)\right\| \leqslant C
$$

for $(\lambda, t) \in M$ and $0 \leqslant s \leqslant t$. By the continuity of $F$ and assumption $\mathrm{H} 4$ there exists $\delta_{2}>0$ such that for $\|\phi\| \leqslant \delta_{2}$ we can assume

$$
\left\|R\left(x_{s}, \lambda\right)\right\| \leqslant\left\|x_{s}\right\|
$$

for $0 \leqslant s \leqslant t$ and all $(\lambda, t) \in M$. (21) then implies that for $\|\phi\| \leqslant \delta_{2}$ and $(\lambda, t) \in M$ we have

$$
\left\|x_{t}\right\| \leqslant C\|\phi\|+C \int_{0}^{t}\left\|x_{s}\right\| d s .
$$

It follows by Gronwall's inequality that there is a constant $C_{1}$ such that for $\|\phi\| \leqslant \delta_{2}, 0 \leqslant s \leqslant t$ and $(\lambda, t) \in M$ we have

$$
\left\|x_{s}\right\| \leqslant C_{1}\|\phi\| \text {. }
$$

Using (21) and (23) and $\mathrm{H} 4$, the result now follows easily.

Next we need to recall the basic result from $\S 8$ of [1]. Let $V$ be a real finite dimensional vector space; denote by $G L(V)$ the invertible linear operators taking $V$ to $V$. It is well known that $G L(V)$ comprises two connected 
components: $G L^{+}(V)$, the component containing the identity $I$ and $G L^{-}(V)$. Suppose that $\Lambda$ is an open interval of real numbers and that for each $\lambda \in \Lambda, M_{\lambda}: V \rightarrow V$ is a linear map. Assume that $\lambda \rightarrow M_{\lambda}$ is continuous. Following [1], suppose that $i \beta(\beta>0)$ is an eigenvalue of $M_{\lambda_{0}}$ and denote by Mult $(i \beta)$ the set $\left\{i m \beta: m=\right.$ a positive integer, im $\beta$ is an eigenvalue of $\left.M_{\lambda_{0}}\right\}$. Assume that 0 is not an eigenvalue of $M_{\lambda_{0}}$. If we cover each element $z$ of Mult $(i \beta)$ by a disc $D_{z}$ which contains only the eigenvalue $z$ of $M_{\lambda_{0}}$, then for $\lambda$ close enough to $\lambda_{0}$, each disc $D_{z}$ contains an eigenvalue $z_{\lambda}$ of $M_{\lambda}$. Furthermore, if the algebraic multiplicity of $z$ as an eigenvalue of $M_{\lambda_{0}}$ is $k$, then $D_{z}$ will contain $k$ eigenvalues of $M_{\lambda}$ (counting algebraic multiplicities) for $\mid \lambda-$ $\lambda_{0} \mid$ small. Denote by $\operatorname{Mult}_{\lambda}(i \beta)$ the set of eigenvalues which lie in the union of the discs $D_{z}$ for $z \in$ Mult $(i \beta)$. Assume that there is an open interval $\Lambda_{0}$ containing $\lambda_{0}$ such that if $\lambda \in \Lambda_{0}-\left\{\lambda_{0}\right\}$ and $z \in \operatorname{Mult}(i \beta)$, then the real part of $z$ is nonzero; by the above discussion we can assume that $\operatorname{Mult}_{\lambda}(i \beta)$ and Mult $(i \beta)$ have the same number of elements (counting algebraic multiplicity) for $\lambda \in \Lambda_{0}$.

With the above assumptions, it makes sense to define $r_{+}$to be the number of elements of $\operatorname{Mult}(i \beta)$ (counting algebraic multiplicity) whose real part is positive for $\lambda>\lambda_{0}$ and $\lambda \in \Lambda_{0}$ and $r_{-}$to be the number of elements of $\operatorname{Mult}_{\lambda}(i \beta)$ whose real part is positive for $\lambda<\lambda_{0}$. Define the index of $i \beta$ with respect to $M_{\lambda}$ to be $r=r_{+}-r_{-}$and the parity of $i \beta$ to be the parity (even or odd) of $r$. Then Alexander and Yorke [1, §8] prove the following lemma.

LEMMA 2 (ALEXANDER-YORKE). Let notation and assumptions be as in the above two paragraphs. Let $t_{0}=2 \pi / \beta$, and for $\rho>0$ define $S_{\rho}=\{(\lambda, t):(\lambda-$ $\left.\lambda_{0}\right)^{2}+\left(t-t_{0}\right)^{2}=\rho^{2}$ \}. Then there exists $\rho_{0}>0$ such that for $0<\rho<\rho_{0}$, the map $(\lambda, t) \in S_{\rho} \rightarrow \exp \left(t M_{\lambda}\right)-I$ is a continuous map into $G L^{ \pm}(V)$. Since $S_{\rho}$ is homotopic to $S^{1}$, the above map gives an element $h$ of $\Pi_{1}\left(G L^{ \pm}(V)\right)$; and if the parity of $i \beta$ is $k$ and $g$ denotes a generator of $\Pi_{1}\left(G L^{ \pm}(V)\right), h=g^{k}$. In particular, $h$ is nonzero if $k$ is odd and $h=g$ if $k$ is odd and $\operatorname{dim}(V)>2$.

We wish to make assumptions analogous to those of the Alexander-Yorke lemma. Thus suppose

H5. The equation det $\Delta_{\lambda_{0}}(z)=0$ has a solution $i \beta$ with $\beta>0$, and no zero solution. There exist positive constants $\varepsilon$ and $M$ such that if det $\Delta_{\lambda}(z)=0$ for $\left|\lambda-\lambda_{0}\right| \leqslant \varepsilon$, then $|\operatorname{Im}(z)| \leqslant M$. Let $\operatorname{Mult}(i \beta)$ denote the set $\{i m \beta: m$ a positive integer and det $\left.\Delta_{\lambda_{0}}(i m \beta)=0\right\}$. Cover the elements $z$ of $\operatorname{Mult}(i \beta)$ by closed discs $D_{z}$ which contain only the eigenvalue $z$ of $A_{\lambda_{0}}$, denote by $D$ the union of the discs $D_{z}$ and let $\operatorname{Mult}_{\lambda}(i \beta)=\left\{z: \operatorname{det} \Delta_{\lambda}(z)=0\right.$ and $\left.z \in D\right\}$. Assume that there is an open interval $\Lambda_{0}$ containing $\lambda_{0}$ such that the real part of $z$ is nonzero if $z \in \operatorname{Mult}_{\lambda}(i \beta)$ and $\lambda \in \Lambda_{0}-\left\{\lambda_{0}\right\}$.

REMARK 3. If $H 5$ holds, then for $\left|\lambda-\lambda_{0}\right|$ small, the number of elements in 
$\operatorname{Mult}_{\lambda}(i \beta)$ (counted with algebraic multiplicity) is the same as the number of elements in $\operatorname{Mult}(i \beta)$ (counted with algebraic multiplicity).

Definition 3. If H5 holds, the index of $i \beta$ with respect to $L_{\lambda}$ is $r=r_{+}-$ $r_{-}$, where $r_{+}\left(r_{-}\right.$, respectively) is the number of elements of Mult $\lambda_{\lambda}(i \beta)$ (counting algebraic multiplicity in the sense of \$2) whose real part is positive for $\lambda>\lambda_{0}\left(\lambda<\lambda_{0}\right.$, respectively). The parity of $i \beta$ is the parity (even or odd) of $r$.

In the statement of the next theorem, the projections $P_{m}$ are as in $\S 1$, and we denote by $X_{m}$ the $m(n+1)$ dimensional range of $P_{m}$ in $X$.

THEOREM 3. Assume that $\mathrm{H} 1-\mathrm{H} 5$ hold and that the pairty of $i \beta$ is odd. Let $t_{0}=2 \pi / \beta$, and for $\rho>0$ define $S_{\rho}=\left\{(\lambda, t):\left(\lambda-\lambda_{0}\right)^{2}+\left(t-t_{0}\right)^{2}=\rho^{2}\right\}$. Then there exist $\rho_{0}>0$ and, for each positive $\varepsilon<\rho_{0}$, an integer $m_{0}=m_{0}(\varepsilon)$ such that for $\varepsilon \leqslant \rho \leqslant \rho_{0}$ and for $m \geqslant m_{0}$, the map $(\lambda, t) \in S_{\rho} \rightarrow I-$ $P_{m} T_{\lambda}(t) \mid X_{m}$ is a continuous map into $G L^{ \pm}\left(X_{m}\right)$ and gives a nonzero element of $\Pi_{1}\left(G L^{ \pm}\left(X_{m}\right)\right)$.

Proof. The proof is long and we divide it into steps.

Step 1. First we show that there is $\rho_{0}>0$ such that $I-T_{\lambda}(t)$ is one-one for $0<\left|\lambda-\lambda_{0}\right|^{2}+\left|t-t_{0}\right|^{2}<\rho_{0}^{2}$. It suffices to show that 1 is not in $\sigma\left(T_{\lambda}(t)\right)$ for such $(\lambda, t)$. By the work of $\S 2$ (since we assume det $\left.\Delta_{\lambda_{0}}(0) \neq 0\right)$ this is equivalent to showing $2 \pi i j / t \notin \sigma\left(A_{\lambda}\right)$ for $(\lambda, t) \in S_{\rho}$ and positive integers $j$. If $\left(\lambda_{0}-\rho_{0}, \lambda_{0}+\rho_{0}\right) \subset \Lambda_{0}, \mathrm{H} 5$ assures that (with possibly a smaller $\left.\rho_{0}\right) \sigma\left(A_{\lambda}\right)$ contains no points ir, $r$ real, for $0<\left|\lambda-\lambda_{0}\right| \leqslant \rho_{0}$. If $\lambda=\lambda_{0}, t=t_{0} \pm \rho$, and one can see that if $\rho_{0}$ is taken small enough, $2 \pi i j / t_{0} \notin \sigma\left(A_{\lambda_{0}}\right)$ for positive integers $j$ and for $0<\rho \leqslant \rho_{0}$. Note that if $0 \in \sigma\left(A_{\lambda_{0}}\right)$, then $I \in \sigma\left(T_{\lambda_{0}}(t)\right)$ for all $t \geqslant 0$, and the above argument fails.

Step 2. Take $\varepsilon>0$ with $0<\varepsilon<\rho_{0}$. We shall show that there exists $m_{0}$ such that for $m \geqslant m_{0}$ and $(\lambda, t) \in S_{\rho}$ with $\varepsilon \leqslant \rho \leqslant \rho_{0}$, the map $I-P_{m} T_{\lambda}(t)$ is one-one. Suppose not. Then there is a sequence $\left(\lambda_{j}, t_{j}\right) \in S_{\rho_{j}}$ and a sequence of integers $m_{j} \rightarrow \infty$ such that $I-P_{m_{j}} T_{\lambda_{j}}\left(t_{j}\right)$ is not one-one; in particular, there is a sequence of unit vectors $\phi_{j}$ with $\phi_{j}-P_{m_{j}} T_{\lambda_{j}}\left(t_{j}\right)\left(\phi_{j}\right)=0$. We can assume $\rho_{0}<t_{0}$ and apply Theorem 2 to the linear system $x^{\prime}(t)=L_{\lambda}\left(x_{t}\right)$; according to Theorem 2 we can assume, by taking a subsequence, that $\phi_{j} \rightarrow \phi, \lambda_{j} \rightarrow \lambda$ and $t_{j} \rightarrow t$. It follows by continuity that $\phi-T_{\lambda}(t)(\phi)=0$, which contradicts the fact that $I-T_{\lambda}(t)$ is one-one.

Since $(\lambda, t) \in S_{\rho} \rightarrow I-P_{m} T(\lambda, t) \mid X_{m}$ is continuous in the norm topology for operators on $X_{m}$ (because $X_{m}$ is finite dimensional), it determines an element of $\Pi_{1}\left(G L^{ \pm}\left(X_{m}\right)\right)$ for $m \geqslant m_{0}$. We now wish to show that this element of the homotopy group is nonzero.

Step 3. As a preliminary step we shall show that the map $(\lambda, t) \rightarrow I-$ $P_{m} T_{\lambda}(t) \mid X_{m}$ is homotopic in $G L^{ \pm}\left(X_{m}\right)$ to a map of the form 


$$
(\lambda, t) \rightarrow I-P_{m} B_{\lambda}^{-1} T_{\lambda}(t) B_{\lambda} P_{\lambda_{0}}-P_{m} C_{\lambda}^{-1} T_{\lambda}(t) C_{\lambda} Q_{\lambda_{0}}
$$

For each element $z_{j}=i k_{j} \beta \in \operatorname{Mult}(i \beta)$ let $\Gamma_{j}$ be a circle about $z_{j}$ which contains no other points of $\sigma\left(A_{\lambda_{0}}\right)$. Let $\bar{\Gamma}_{j}$ be a circle about $-i k_{j} \beta$ and denote by $\Gamma$ the union of these circles. As in $\$ 2$, for $\lambda$ near $\lambda_{0}$ define

$$
P_{\lambda}=\frac{1}{2 \pi i} \int_{\Gamma}\left(z-A_{\lambda}\right)^{-1} d z .
$$

By Proposition 2, $\S 2, P_{\lambda}$ maps the real $B$-space $X$ into $X$. We follow the notation of $\S 2$, so $X_{\lambda}$ is the range of $P_{\lambda}$, etc. For $\left|\lambda-\lambda_{0}\right|<\rho_{0}$ we can assume that $\left\|P_{\lambda}-P_{\lambda_{0}}\right\|<1$, and it follows that for such $\lambda$ that

$$
B_{\lambda}=I+\left(P_{\lambda}-P_{\lambda_{0}}\right) \text { and } C_{\lambda}=I+\left(Q_{\lambda}-Q_{\lambda_{0}}\right)
$$

are one-one and onto $X$. Since $B_{\lambda}\left(X_{\lambda_{0}}\right) \subset X_{\lambda}$, and since $X_{\lambda}$ and $X_{\lambda_{0}}$ have the same dimension, we get $B_{\lambda}\left(X_{\lambda_{0}}\right)=X_{\lambda}$ and $B_{\lambda}^{-1}\left(X_{\lambda}\right)=X_{\lambda_{0}}$. A slightly more involved argument (which we omit) shows that $C_{\lambda}\left(Y_{\lambda_{0}}\right)=Y_{\lambda}$ and $C_{\lambda}^{-1}\left(Y_{\lambda}\right)=$ $Y_{\lambda_{0}}$

Now assume that $\rho_{0}$ is small, and for $0<\rho \leqslant \rho_{0}$ and each real number $\mu$ with $0 \leqslant \mu<1$ define $q_{\mu}: S_{\rho} \rightarrow L(X, X)=$ bounded linear operators on $X$,

$$
q_{\mu}(\lambda, t)=I-B_{\mu(\lambda)}^{-1} T_{\lambda}(t) B_{\mu(\lambda)} P_{\lambda_{0}}-C_{\mu(\lambda)}^{-1} T_{\lambda}(t) C_{\mu(\lambda)} Q_{\lambda_{0}},
$$

where $\mu(\lambda)=(1-\mu) \lambda+\mu \lambda_{0}$. Note that for $\mu=1$ we obtain $I-T_{\lambda}(t)$. We claim that the linear operator $q_{\mu}(\lambda, t)$ is one-one for $\mu, \lambda, t$ as above. To see this suppose

$$
q_{\mu}(\lambda, t)(\phi)=0
$$

for a nonzero $\phi$. Then composing on the left with $P_{\lambda_{0}}$ and $Q_{\lambda_{0}}$, respectively, gives

$$
\begin{aligned}
& P_{\lambda_{0}} \phi-B_{\mu}^{-1} T_{\lambda}(t) B_{\mu} P_{\lambda_{0}} \phi=0, \\
& Q_{\lambda_{0}} \phi-C_{\mu}^{-1} T_{\lambda}(t) C_{\mu} Q_{\lambda_{0}} \phi=0 .
\end{aligned}
$$

From (26) we derive that

$$
\left(I-T_{\lambda}(t)\right) B_{\mu} P_{\lambda_{0}} \phi=0, \quad\left(I-T_{\lambda}(t)\right) C_{\mu} Q_{\lambda_{0}} \phi=0 .
$$

Since $I-T_{\lambda}(t)$ is one-one for $(\lambda, t) \in S_{\rho}$, and because $B_{\mu}$ and $C_{\mu}$ are one-one, equations (27) imply that $\phi=0$.

Next suppose that $\varepsilon$ is any positive number with $\varepsilon<\rho_{0}$. We claim that there is an integer $N=N(\varepsilon)$ such that for $m \geqslant N, \varepsilon \leqslant \rho \leqslant \rho_{0}$ and $(\lambda, t) \in S_{\rho}$ the linear operator

$$
I+P_{m}\left(q_{\mu}(\lambda, t)-I\right)
$$

is one-one.

As in previous cases, the proof is by contradiction. Suppose not. Then there is a sequence of integers $m(j) \rightarrow \infty$, a sequence of unit vectors $\phi_{j} \in X$ and 
sequences $\left(\lambda_{j}, t_{j}\right) \in S_{\rho_{j}}$ and $\mu_{j} \in[0,1]$ for which, when $\mu=\mu_{j}, \lambda=\lambda_{j}, t=t_{j}$ and $m=m(j)$, the operator in (28) vanishes when applied to $\phi_{j}$. We can assume by taking subsequences that $\left(\lambda_{j}, t_{j}\right) \rightarrow(\lambda, t) \in S_{\rho}$ and $\mu_{j} \rightarrow \mu$. Notice that $B_{\mu\left(\lambda_{j}\right)}^{-1}$ approaches $B_{\mu(\lambda)}^{-1}$ in norm and that $B_{\mu(\lambda)}^{-1}=I+K$, where $K$ is a compact linear operator; analogous statements hold for $C_{B_{3}\left(\lambda_{3}\right)}^{-1}$. It follows from the compactness of $K$ that by taking a further subsequence we can assume

$$
q_{\mu j}\left(\lambda_{j}, t_{j}\right)\left(\phi_{j}\right)-\left(I-T_{\lambda_{j}}\left(t_{j}\right) B_{\mu} P_{\lambda_{0}}-T_{\lambda_{j}}\left(t_{j}\right) C_{\mu} Q_{\lambda_{0}}\right)\left(\phi_{j}\right)
$$

converges in norm to some element $\psi$. Using this fact, we can assume that

$$
\phi_{j}-P_{m(j)} T_{\lambda_{j}}\left(t_{j}\right) B_{\mu_{j}} P_{\lambda_{0}}\left(\phi_{j}\right)-P_{m(j)} T_{\lambda_{j}}\left(t_{j}\right) C_{\mu_{j}} Q_{\lambda_{0}}\left(\phi_{j}\right)
$$

converges in norm as $j \rightarrow \infty$. Since $B_{\mu}$ and $C_{\mu}$ approach $B_{\mu}$ and $C_{\mu}$ in norm and $B_{\mu}$ and $C_{\mu}$ are compact perturbations of the identity, we can assume by taking a further subsequence that

$$
\phi_{j}-P_{m(j)} T_{\lambda_{j}}\left(t_{j}\right) \phi_{j}
$$

converges in norm as $j \rightarrow \infty$. It now follows from Theorem 2 that by taking a still further subsequence we can assume that

$$
\phi_{j} \rightarrow \phi \text {. }
$$

It now follows from (28) by taking limits that

$$
\left(q_{\mu}(\lambda, t)\right)(\phi)=0
$$

and this contradicts the previous results.

We have shown that for $m \geqslant N$, (28) provides a permissible homotopy in $G L^{ \pm}\left(X_{m}\right)$ bctween $I-P_{m} T_{\lambda}(t) \mid X_{m}$ and $P_{m} q_{1}(\lambda, t) \mid X_{m}$.

Step 4. We shall now show that for $m$ large enough there is a homotopy in $G L^{ \pm}\left(X_{m}\right)$ between the map $(\lambda, t) \rightarrow P_{m} q_{1}(\lambda, t) \mid X_{m}$ and the map

$$
(\lambda, t) \rightarrow I-P_{m} B_{\lambda}^{-1} T_{\lambda}(t) B_{\lambda} P_{\lambda_{0}}-P_{m} T_{\lambda_{0}}\left(t_{0}\right) Q_{\lambda_{0}} \mid X_{m}
$$

If we recall that $I-T_{\lambda}(t) \mid Y_{\lambda_{0}}$ (where $Y_{\lambda_{0}}$ is the range of $Q_{\lambda_{0}}$ ) is one-one and onto $Y_{\lambda_{0}}$ for $\left(\lambda-\lambda_{0}\right)^{2}+\left(t-t_{0}\right)^{2} \leqslant \rho_{0}^{2}$, it is not hard to see that if we define $s(\lambda)=(1-s) \lambda+s \lambda_{0}$ and $s(t)=(1-s) t+s t_{0}$ for $0 \leqslant s \leqslant 1$, then

$$
H_{s}(\lambda, t)=I-B_{\lambda}^{-1} T_{\lambda}(t) B_{\lambda} P_{\lambda_{0}}-C_{s(\lambda)}^{-1} T_{s(\lambda)}(s(t)) C_{s(\lambda)} Q_{\lambda_{0}}
$$

is one-one for $0 \leqslant s \leqslant 1$ and for $0<\varepsilon \leqslant\left(\lambda-\lambda_{0}\right)^{2}+\left(t-t_{0}\right)^{2} \leqslant \rho_{0}^{2}$. Arguments similar to ones previously used show that for $m \geqslant N_{1}=N_{1}(\varepsilon)$,

$$
P_{m} H_{s}(\lambda, t) \mid X_{m}
$$

is one-one for $0 \leqslant s \leqslant 1$ and $(\lambda, t)$ as above, and this establishes the assertion in Step 4.

The homotopies of Steps 3 and 4 reduce the problem to showing that

$$
(\lambda, t) \rightarrow I-P_{m} B_{\lambda}^{-1} T_{\lambda}(t) B_{\lambda} P_{\lambda_{0}}-P_{m} T_{\lambda_{0}}\left(t_{0}\right) Q_{\lambda_{0}} \mid X_{m}
$$


gives a nonzero element of $\Pi_{1}\left(G L^{ \pm}\left(X_{m}\right)\right)$ for $m$ large.

Step 5. The form of (36) is still not quite suitable. In this step we shall show that for $(\lambda, t) \in S_{\rho}, 0<\varepsilon \leqslant \rho \leqslant \rho_{0}, 0 \leqslant s \leqslant 1$, and $m$ large enough the linear operator

$$
I-\left(s P_{m}+(1-s) I\right) B_{\lambda}^{-1} T_{\lambda}(t) B_{\lambda} P_{\lambda_{0}}-P_{m} T_{\lambda_{0}}\left(t_{0}\right) Q_{\lambda_{0}}
$$

is one-one as a map of $X$ to $X$.

To prove this, first observe that

$$
G(\lambda, t)=I-B_{\lambda}^{-1} T_{\lambda}(t) B_{\lambda} P_{\lambda_{0}}-T_{\lambda_{0}}\left(t_{0}\right) Q_{\lambda_{0}}
$$

is one-one and onto $X$ for $(\lambda, t) \in S_{\rho}$ and $0<\rho<\rho_{0}$. Furthermore, because $P_{\lambda_{0}}$ has finite dimensional range, the map

$$
(\lambda, t) \rightarrow G(\lambda, t)
$$

is continuous in the norm operator topology, not just the strong operator topology. It follows that there exists a positive constant $c=c(\varepsilon)$ such that for any $\rho$ with $0<\varepsilon \leqslant \rho \leqslant \rho_{0}$ and any unit vector $\phi$ in $X$,

$$
\left\|\phi-B_{\lambda}^{-1} T_{\lambda}(t) B_{\lambda} P_{\lambda_{0}}(\phi)-T_{\lambda_{0}}\left(t_{0}\right) Q_{\lambda_{0}}(\phi)\right\|>c .
$$

An argument using the strong $A$-properness of $I-T_{\lambda_{0}}\left(t_{0}\right)$ now shows that for $m$ large enough and $\phi$ and $(\lambda, t)$ as above, we have

$$
\left\|\phi-P_{m} B_{\lambda}^{-1} T_{\lambda}(t) B_{\lambda} P_{\lambda_{0}}(\phi)-P_{m} T_{\lambda_{0}}\left(t_{0}\right) Q_{\lambda_{0}}(\phi)\right\|>\frac{c}{2} .
$$

Using the fact that $P_{\lambda_{0}}$ is a compact linear operator, it is not hard to show that for $m$ large enough and $\left(\lambda-\lambda_{0}\right)^{2}+\left(t-t_{0}\right)^{2}<\rho_{0}^{2}$, we have

$$
\left\|P_{m} B_{\lambda}^{-1} T_{\lambda}(t) B_{\lambda} P_{\lambda_{0}}-B_{\lambda}^{-1} T_{\lambda}(t) B_{\lambda} P_{\lambda_{0}}\right\| \leqslant c / 4 \text {. }
$$

It follows that for $m$ large, $0<\varepsilon \leqslant \rho \leqslant \rho_{0},(\lambda, t) \in S_{\rho}$ and $0 \leqslant \rho \leqslant 1$, the following operator is one-one:

$$
I-\left(s P_{m}+(1-s) I\right) B_{\lambda}^{-1} T_{\lambda}(t) B_{\lambda} P_{\lambda_{0}}-P_{m} T_{\lambda_{0}}\left(t_{0}\right) Q_{\lambda_{0}}
$$

Step 6. Now let $Y_{m}$ denote the linear subspace spanned by $X_{m}$ and $X_{\lambda_{0}}$. To show that the map given by (36) yields a nonzero element of $\Pi_{1}\left(G L^{ \pm}\left(X_{m}\right)\right)$, it suffices to show that

$$
(\lambda, t) \rightarrow I-P_{m} B_{\lambda}^{-1} T_{\lambda}(t) B_{\lambda} P_{\lambda_{0}}-P_{m} T_{\lambda_{0}}\left(t_{0}\right) Q_{\lambda_{0}} \mid Y_{m}
$$

gives a nonzero element of $\Pi_{1}\left(G L^{ \pm}\left(Y_{m}\right)\right)$. (42) gives a homotopy in $G L^{ \pm}\left(Y_{m}\right)$ for $0 \leqslant s \leqslant 1$, so it suffices to show that

$$
(\lambda, t) \rightarrow I-B_{\lambda}^{-1} T_{\lambda}(t) B_{\lambda} P_{\lambda_{0}}-P_{m} T_{\lambda_{0}}\left(t_{0}\right) Q_{\lambda_{0}} \mid Y_{m}
$$

gives a nonzero element of $\Pi_{1}\left(G L^{ \pm}\left(Y_{m}\right)\right)$. If we note that $Q_{\lambda_{0}}\left(Y_{m}\right) \subset Y_{m}$, and if we write

$$
Y_{m}=X_{\lambda_{0}} \oplus Q_{\lambda_{0}}\left(Y_{m}\right)
$$


then the map in (44) is reduced by $X_{\lambda_{0}}$ and $Q_{\lambda_{0}}\left(Y_{m}\right)$. On $X_{\lambda_{0}}$ the map is given by

$$
(\lambda, t) \rightarrow I-B_{\lambda}^{-1} T_{\lambda}(t) B_{\lambda} \mid X_{\lambda_{0}},
$$

and on $\left(Q_{\lambda_{0}}\left(Y_{m}\right)\right)$ it is given by

$$
(\lambda, t) \rightarrow I-P_{m} T_{\lambda_{0}}\left(t_{0}\right) \mid Q_{\lambda_{0}}\left(Y_{m}\right)
$$

which is just a constant map. It follows that to show (44) gives a nonzero element, it suffices to show the map (46) is $g^{r}$ in $\Pi_{1}\left(G L^{ \pm}\left(X_{\lambda_{0}}\right)\right)$, where $r$ is the index of $i \beta$ and $g$ is a generator of $\Pi_{1}\left(G L^{ \pm}\left(X_{\lambda_{0}}\right)\right)$. Notice that $A_{\lambda}$, the infinitesimal generator of $T_{\lambda}(t)$, is everywhere defined on $X_{\lambda}$, so that we have

$$
T_{\lambda}(t) \mid X_{\lambda}=\sum_{j=0}^{\infty} \frac{\left(t A_{\lambda}\right)^{j}}{j !} .
$$

Thus we find that if we set $M_{\lambda}=B_{\lambda}^{-1} A_{\lambda} B_{\lambda} \mid X_{\lambda_{0}}$, we have

$$
B_{\lambda}^{-1} T_{\lambda}(t) B_{\lambda} \mid X_{\lambda_{0}}=\exp \left(t M_{\lambda}\right) \text {. }
$$

If $z \in \operatorname{Mult}_{\lambda}(i \beta)$, the results of $\$ 2$ show that the algebraic multiplicity of $z$ as an element of the spectrum of $A_{\lambda}$ is the same as the multiplicity of $z$ as a root of $\operatorname{det}\left(x-A_{\lambda} \mid X_{\lambda}\right)$, which in turn is the multiplicity of $z$ as a root of $\operatorname{det}\left(x-M_{\lambda}\right)$. This shows that the index of $i \beta$ in our theorem is the same as the index of $i \beta$ with respect to $M_{\lambda}$. Thus the Alexander-Yorke theorem implies that (46) gives a nonzero element of $\Pi_{1}\left(G L^{ \pm}\left(X_{\lambda_{0}}\right)\right)$, and we are done.

4. Global bifurcation of periodic solutions of retarded functional differential equations. After the preliminaries of the previous three sections, we can begin to prove our main theorem. Assuming that $\mathrm{H} 1-\mathrm{H} 3$ hold, we write

$$
\begin{gathered}
S=\{(\phi, \lambda, t) \in X \times \Omega \times[0, \infty): x(s ; \phi, \lambda) \text { is periodic } \\
\text { of period } t>0 \text { (not necessarily least period) } \\
\text { and } x(s) \text { is not a constant function }\} . \\
\delta=\text { the closure of } S \text { in } X \times \Lambda \times[0, \infty) .
\end{gathered}
$$

LEMMA 3. Assume H1-H3. If $(\phi, \lambda, t)$ is an element of $\mathcal{S}$ but not of $S$, then $\phi$ is a constant function and $f(\phi, \lambda)=0$; in particular, $x(s ; \phi, \lambda)$ is a constant function.

Proof. By definition, there exists a sequence $\left(\phi_{j}, \lambda_{j}, t_{j}\right) \in S$ which converges to $(\phi, \lambda, t)$. If we define

$$
x_{j}(s)=x\left(s ; \phi_{j}, \lambda_{j}\right)
$$

$x_{j}(s)$ is periodic with period $t_{j}, x_{j}[-\gamma, 0]=\phi_{j}$ and 


$$
x_{j}(u)=\phi_{j}(0)+\int_{0}^{u} f\left(\left(x_{j}\right)_{s}, \lambda_{j}\right) d s
$$

for $u \geqslant 0 . \mathrm{H} 3$ and the periodicity of $x_{j}$ imply that there is a constant $M_{1}$ such that

$$
\left|x_{j}(s)\right| \leqslant M_{1}
$$

for $j \geqslant 1$ and $s \geqslant-\gamma$. (52) and the fact that $f$ is bounded on bounded sets now imply that the functions $x_{j}$ are Lipschitzian with uniform Lipschitz constant $M_{2}$. The functions $x_{j}[-\gamma, t+1]$ form a bounded, equicontinuous family, so by taking a subsequence we can assume that $x_{j}$ approaches some continuous function $y$ uniformly on $[-\gamma, t+1]$, and by continuity we see that $y$ satisfies

$$
\begin{gathered}
y(u)=\phi(0)+\int_{0}^{u} f\left(y_{s}, \lambda\right) d s \text { for } u \geqslant 0, \\
y \mid[-\gamma, 0]=\phi .
\end{gathered}
$$

It follows that $y(s)=x(s ; \phi, \lambda)$ for $s \geqslant-\gamma$. There are two cases to consider.

(i) $t>0$. We know by equicontinuity and the facts that $t_{j} \rightarrow t$ and $x_{j}$ has period $t_{j}$ that

$$
\lim _{j \rightarrow \infty}\left(\sup _{-\gamma<s<0}\left|x_{j}(t+s)-x_{j}(s)\right|\right)=0 .
$$

(55) implies that $y(t+s)=y(s)$ for $-\gamma \leqslant s \leqslant 0$, so $y(s)=x(s ; \phi, \lambda)$ is periodic of period $t>0$. Since we assume that $(\phi, \lambda, t) \notin S$, we must have that $y(s)$ is a constant function.

(ii) $t=0$. Since $x_{j}$ is periodic of period $t_{j}$ and Lipschitzian with Lipschitz constant $M_{2}$ (independent of $j$ ), we have for any numbers $s_{1}, s_{2} \geqslant-\gamma$ that

$$
\left|x_{j}\left(s_{1}\right)-x_{j}\left(s_{2}\right)\right| \leqslant M_{2} t_{j} \text {. }
$$

By taking limits (since $t_{j} \rightarrow 0$ ) we obtain that $x(s ; \phi, \lambda)$ is a constant function.

Before proving our next lemma, we need to recall some facts from homotopy theory. Let notation be as in $\S 3$, so that for $\rho>0, S_{\rho} \subset \mathbf{R}^{2}$ is a homeomorphic image of a circle. Suppose that $(\lambda, t) \in S_{\rho} \rightarrow A(\lambda, t) \in$ $\mathrm{GL}^{+}(n)$ is a continuous map. For $r>0$ define (following [13]) $S_{r, \rho}$ by

$$
\begin{aligned}
S_{r, \rho}= & \left\{(x, \lambda, t): x \in \mathbf{R}^{n}, \lambda, t \in \mathbf{R},\right. \\
& \left.\|x\|^{2}+\left|\lambda-\lambda_{0}\right|^{2}+\left|t-t_{0}\right|^{2}=r^{2}+\rho^{2}\right\} .
\end{aligned}
$$

Of course, $S_{r, \rho}$ is homeomorphic to $S^{n+1}$, the $n+1$ sphere. Extend the map $(\lambda, t) \rightarrow A(\lambda, t)$ to all of $\mathbf{R}^{2}$ in any way such that $A(\lambda, t)$ is nonsingular for $(\lambda, t) \neq\left(\lambda_{0}, t_{0}\right)$. Then we can define a map from $S_{r, \rho}$ to $\mathbf{R}^{n+1}-\{0\}$ by

$$
(x, \lambda, t) \rightarrow\left(A(\lambda, t) x,\|x\|^{2}-r^{2}\right) .
$$


If we use the notation $\left[Y_{1}, Y_{2}\right]$ to denote the free homotopy classes of continuous maps from the topological space $Y_{1}$ to the topological space $Y_{2}$, then (57) induces a map $J$ from $\left[S^{1}, G L^{+}(n)\right]$ to $\left[S_{r, \rho}, \mathbf{R}^{n+1}-\{0\}\right]$, and the map $J$ is a bijection. Further details and references can be found in [12].

In our case, if we define

$$
\begin{aligned}
S_{r, \rho}(m)= & \left\{(x, \lambda, t) \in X_{m} \times \mathbf{R}^{2}:\right. \\
& \left.\|x\|^{2}+\left(\lambda-\lambda_{0}\right)^{2}+\left(t-t_{0}\right)^{2}=r^{2}+\rho^{2}\right\}
\end{aligned}
$$

and assume that $0<r, \rho$ and $\rho \leqslant \rho_{0}$, then Theorem 3 of $\$ 3$ in conjunction with the above remarks shows that the map

$$
\begin{aligned}
(x, \lambda, t) \in S_{r, \rho}(m) & \rightarrow\left(x-P_{m} T_{\lambda}(t) x,\|x\|^{2}-r^{2}\right) \\
& \in\left(X_{m} \times \mathbf{R}\right)-\{0\}
\end{aligned}
$$

defines (for $m$ large enough) a nontrivial element of $\left[S_{r, \rho}(m),\left(X_{m} \times \mathbf{R}\right)-\right.$ $\{0\}]$ (that is, the map in (59) is not homotopic to the constant map).

With these preliminaries we can study the homotopy type of the map

$$
(x, \lambda, t) \rightarrow\left(x-P_{m} F(x, \lambda, t),\|x\|^{2}-r^{2}\right),
$$

where $F$ is defined by $(7)$ in $\S 1$.

Lemma 4. Assume H1-H5 and that the parity of $i \beta$ is odd (see Definition 3 in §3). Let $\rho \leqslant \rho_{0}$ be a positive number. Then there exists a positive number $r(\rho)$ (depending continuously on $\rho$ ) such that if $0<r<r(\rho)$ and $m \geqslant m(r)$, (60) defines a map from $S_{r, \rho}(m)$ to $\left(X_{m} \times \mathbf{R}\right)-\{0\}$ which is not homotopic to a constant map.

Proof. Since $I-T_{\lambda}(t)$ depends continuously on $(\lambda, t)$ and is one-one and onto for $\left(\lambda-\lambda_{0}\right)^{2}+\left(t-t_{0}\right)^{2}=\rho^{2}, 0<\rho \leqslant \rho_{0}$, there is a continuous positive function $c(\rho)$ such that

$$
\left\|x-T_{\lambda}(t) x\right\| \geqslant c(\rho)\|x\|
$$

for $(\lambda, t)$ as above. Furthermore, it is not hard to show that there is a continuous, strictly increasing function $\varepsilon(r)$ of $r$ for $r \geqslant 0$ with $\varepsilon(0)=0$ such that for all $(\lambda, t)$ with $\left(\lambda-\lambda_{0}\right)^{2}+\left(t-t_{0}\right)^{2} \leqslant \rho_{0}^{2}$ we have

$$
\left\|T_{\lambda}(t)(x)-F(x, \lambda, t)\right\| \leqslant \varepsilon(\|x\|)\|x\| .
$$

If $\varepsilon^{-1}$ denotes the inverse function and we take $0<r \leqslant r(\rho)=\varepsilon^{-1}\left(\frac{1}{2} c(\rho)\right)$, we find that for $\left(\lambda-\lambda_{0}\right)^{2}+\left(t-t_{0}\right)^{2}=\rho^{2}, 0 \leqslant s \leqslant 1$ and $\|x\| \leqslant r$ we have

$$
\left\|x-s T_{\lambda}(t)(x)-(1-s) F(x, \lambda, t)\right\| \geqslant \frac{1}{2} c(\rho)\|x\| .
$$

It follows that if we define, for $0 \leqslant s \leqslant 1$,

$$
\Phi(x, \lambda, t, s)=s T_{\lambda}(t)(x)+(1-s) F(x, \lambda, t),
$$

and let 


$$
\begin{aligned}
\Sigma_{r, \rho}=\{( & (x, \lambda, t) \in X \times \Lambda \times \mathbf{R}^{+}: \\
& \left.\|x\|^{2}+\left(\lambda-\lambda_{0}\right)^{2}+\left(t-t_{0}\right)^{2}=r^{2}+\rho^{2}\right\}
\end{aligned}
$$

(with $r$ and $\rho$ as above), then the map

$$
(x, \lambda, t, s) \in \Sigma_{r, \rho} \times[0,1] \rightarrow\left(x-\Phi(x, \lambda, t, s),\|x\|^{2}-r^{2}\right)
$$

does not have zero in its range.

We now claim that for $m \geqslant m(r)$ the map

$$
(x, \lambda, t, s) \in \Sigma_{r, \rho} \times[0,1] \rightarrow\left(x-P_{m} \Phi(x, \lambda, t, s),\|x\|^{2}-r^{2}\right)
$$

does not have zero in its range. In order to prove this, suppose not, so that there is a sequence $\left(x_{m}, \lambda_{m}, t_{m}, s_{m}\right) \in \Sigma_{r, \rho} \times[0,1]$ for which the right-hand side of (63) vanishes. One can check that $\Phi$ satisfies the hypotheses of Theorem 1, so that by taking a subsequence we can assume that

$$
\left(x_{m}, \lambda_{m}, t_{m}, s_{m}\right) \rightarrow(x, \lambda, t, s) \in \Sigma_{r, \rho} \times[0,1] .
$$

By continuity we have that

$$
\left(x-\Phi(x, \lambda, t, s),\|x\|^{2}-r^{2}\right)=0,
$$

which is a contradiction.

To complete the proof recall that by the results of $\$ 3$, the map

$$
(\lambda, t) \in S_{\rho} \rightarrow I-P_{m} T_{\lambda}(t) \in G L^{ \pm}\left(X_{m}\right)
$$

gives a nonzero element of $\Pi_{1}\left(G L^{ \pm}\left(X_{m}\right)\right)$ for large $m$. By our previous remarks, it follows that for $r, \rho$ as above,

$$
\begin{aligned}
(x, \lambda, t) \in S_{r, \rho}(m) & \rightarrow\left(x-P_{m} T_{\lambda}(t)(x),\|x\|^{2}-r^{2}\right) \\
& \in\left(X_{m} \times \mathbf{R}\right)-\{0\}
\end{aligned}
$$

is not homotopic to a constant in $\left(X_{m} \times \mathbf{R}\right)-\{0\}$. Since we have shown that this map is homotopic in $\left(X_{m} \times \mathbf{R}\right)-\{0\}$ to the map of (60), the lemma is proved.

Now let $\lambda_{0}$ and $t_{0}=2 \pi / \beta$ be as in $\S 3$. We are interested in the connected component $\delta_{0}$ of $\delta$ which contains $\left(0, \lambda_{0}, t_{0}\right) \in X \times \Lambda \times[0, \infty)$.

Lemma 5. Assume that $\mathrm{H} 1-\mathrm{H} 5$ hold and that the pairty of i $\mathrm{i}$ is odd (see Definition 3). If $\delta_{0}$ is defined as above, $\delta_{0}$ is nonempty.

Proof. In the notation of the previous lemma, select a positive number $r$ with $r \leqslant r_{0}=r\left(\rho_{0}\right)$. According to the previous lemma, the map given by (60) is not homotopic to a constant in $\left(X_{m} \times \mathbf{R}\right)-\{0\}$. It follows (see [20, p. 1]) that for all $m$ sufficiently large, there exists $\left(x_{m}, \lambda_{m}, t_{m}\right)$ such that

$$
\begin{gathered}
\left\|x_{m}\right\|^{2}+\left(\lambda_{m}-\lambda_{0}\right)^{2}+\left(t_{m}-t_{0}\right)^{2}<r^{2}+\rho_{0}^{2}, \\
\left\|x_{m}\right\|=r, \quad x_{m}-P_{m} F\left(x_{m}, \lambda_{m}, t_{m}\right)=0 .
\end{gathered}
$$


According to Theorem 2, we can assume by taking a subsequence that $\left(x_{m}, \lambda_{m}, t_{m}\right) \rightarrow(x, \lambda, t)$, and by continuity we obtain

This completes the proof.

$$
\|x\|=r \text { and } x-F(x, \lambda, t)=0 .
$$

We need to recall at this point the definition and basic properties of "compact vector fields"; those concepts have been extensively used by A. Granas [9]. If $Y$ is a Banach space, $A$ is a closed subset of $Y$ and $g: A \rightarrow Y$ is a continuous map, $g$ is a "compact vector field" if $g(x)=x-G(x)$, where $G$ takes bounded sets to precompact sets. We work in the category of compact vector fields; in particular, two compact vector fields $g_{0}$ and $g_{1}$ are (compactly) homotopic if there is a compact map $G: A \times[0,1] \rightarrow X$ such that $g_{0}(x)=x-G(x, 0)$ and $g_{1}(x)=x-G(x, 1)$.

If $B$ is a closed set containing $A$ and $g: A \rightarrow Y-\{0\}=P$ is a compact vector field, we say " $g$ is inessential with respect to $B$ " if $g$ has an extension to a zero field $\bar{g}: B \rightarrow P$. If $g_{0}, g_{1}: A \rightarrow P$ are compact vector fields and if tiney are (compactly) homotopic on $A$ by a zero-free homotopy $x-G(x, t)$ $\in P$, then the basic lemma (see [9]) is that (assuming $B$ is bounded) $g_{0}$ is essential with respect to $B$ if and only if $g_{1}$ is. Of course, in the finite dimensional case more general versions of this lemma have long been known: see [2, Theorem 8.1, p. 94].

We shall need a simple extension of Granas' idea. If $Y$ is a Banach space, $A$ is a closed subset of $Y \times \mathbf{R}^{m}$ and $g: A \rightarrow Y \times \mathbf{R}^{k}$ is a continuous map, we shall say $g$ is a compact vector field if we have $g(y, u)=(y-G(y, u)$, $h(y, u)$ ), where $G$ takes bounded subsets of $A$ into precompact sets and $h$ : $A \rightarrow \mathbf{R}^{k}$ takes bounded sets to bounded sets. If $B$ is a closed, bounded set containing $A$ and $g: A \rightarrow P=\left(Y \times \mathbf{R}^{k}\right)-\{0\}$ is a compact vector field, we say $g$ is inessential with respect to $B$ if $g$ has an extension to a compact vector field $\bar{g}: B \rightarrow P$; otherwise $g$ is "essential with respect to $B$ ". Again, if $B$ is bounded and $g_{0}, g_{1}: A \rightarrow P$ are compact vector fields which are homotopic (in the sense of compact vector fields) by a zero-free homotopy, then $g_{0}$ is essential with respect to $B$ if and only if $g_{1}$ is. The proof is the same as in Granas' case, and we omit it.

With these preliminaries we can prove an extension of Lemma 4.

LemMa 6. Assume that $\mathrm{H} 1-\mathrm{H} 5$ hold and that the parity of i $\beta$ (see Definition 3) is odd. Let $\rho \leqslant \rho_{0}$ and $r \leqslant r(\rho)(r(\rho)$ as in Lemma 4) be positive numbers and define sets $A$ and $B$ by

$$
\begin{aligned}
A=\Sigma(r, \rho)=\{ & (x, \lambda, t) \in X \times \mathbf{R}^{2}: \\
& \left.\|x\|^{2}+\left(\lambda-\lambda_{0}\right)^{2}+\left(t-t_{0}\right)^{2}=r^{2}+\rho^{2}\right\}, \\
B=B(r, \rho)= & \left\{(x, \lambda, t) \in X \times \mathbf{R}^{2}:\right. \\
& \left.\|x\|^{2}+\left(\lambda-\lambda_{0}\right)^{2}+\left(t-t_{0}\right)^{2} \leqslant r^{2}+\rho^{2}\right\} .
\end{aligned}
$$


Then for $n$ large enough the map

(66) $(x, \lambda, t) \in A \rightarrow\left(x-P_{n} F(x, \lambda, t),\|x\|^{2}-r^{2}\right) \in(X \times \mathbf{R})-\{0\}$

is essential with respect to $B$.

Proof. Our first claim is that for $\|x\|=r, 0<s<1$ and $n>n_{0}$ we have

$$
x-s F(x, \lambda, t)-(1-s) P_{n} F(x, \lambda, t) \neq 0 .
$$

If not, there would be a sequence $\left(x_{n_{i}}, \lambda_{n_{i}}, t_{n_{i}}\right)$ for which (67) would be zero. By arguments like those in $\$ 1$, we could then assume (by taking a subsequence) that $\left(x_{n_{i}}, \lambda_{n_{i}}, t_{n_{i}}\right) \rightarrow(x, \lambda, t)$, and we would have $x-F(x, \lambda, t)$ $=0$, contrary to our previous work.

Using the above fact, it follows from Theorem 1 of $\$ 1$ that for any given $n \geqslant n_{0}$, for $\|x\|=r$ and for $0 \leqslant s \leqslant 1$ we have

$$
x-s P_{m} F(x, \lambda, t)-(1-s) P_{n} F(x, \lambda, t) \neq 0
$$

for $m \geqslant m_{0}\left(m_{0}\right.$ dependent on $\left.n\right)$.

To prove the lemma, suppose the contrary, so the map (66) has an extension

$$
(x, \lambda, t) \in B \rightarrow(x-G(x, \lambda, t), h(x, \lambda, t)) \in(X \times \mathbf{R})-\{0\} .
$$

By the compactness of $G$ and $h$ it follows that for $m$ large enough we have

$$
\left(x-P_{m} G(x, \lambda, t), h(x, \lambda, t)\right) \in(X \times \mathbf{R})-\{0\} .
$$

If we take $m$ also to be an integral multiple of $n$, so that $P_{m} P_{n}=P_{n}$, we see from (70) that

$$
\begin{aligned}
(x, \lambda, t) \in A \cap\left(X_{m} \times \mathbf{R}^{2}\right) & \rightarrow\left(x-P_{n} F(x, \lambda, t),\|x\|^{2}-r^{2}\right) \\
& \in\left(X_{m} \times \mathbf{R}\right)-\{0\}
\end{aligned}
$$

is inessential with respect to $B \cap\left(X_{m} \times \mathbf{R}^{2}\right)$. However, our previous work shows that (71) is homotopic to

$$
(x, \lambda, t) \in S_{r, \rho}(m) \rightarrow\left(x-P_{m} F(x, \lambda, t),\|x\|^{2}-r^{2}\right)
$$

(as maps into $\left.\left(X_{m} \times \mathbf{R}\right)-\{0\}\right)$. This shows that (72) is inessential with respect to $B \cap\left(X_{m} \times \mathbf{R}^{2}\right)$, which contradicts the fact that (72) is not homotopic to a constant map.

We can now state and begin to prove our main theorem.

THEOREM 4. Assume that H1-H5 hold and that the parity of $i \beta$ is odd (see Definition $3, \S 3)$. Let $\mathcal{S}$ be defined by (50) and let $\mathcal{S}_{0}$ denote the connnected component of $\mathcal{S}$ which contains $\left(0, \lambda_{0}, t_{0}\right) \in X \times \Lambda \times[0, \infty)$ (Lemma 5 shows that $\delta_{0}$ is nonempty). Then it follows that either

(i) $S_{0}$ is not a compact subset of $X \times \Lambda \times[0, \infty)$, or

(ii) $\delta_{0}$ contains an element of the form $\left(\phi_{1}, \lambda_{1}, t_{1}\right)$, where $\phi_{1}$ is a constant 
function, $f\left(\phi_{1}, \lambda_{1}\right)=0$ and $\left(\phi_{1}, \lambda_{1}, t_{1}\right) \neq\left(0, \lambda_{0}, t_{0}\right)$ (so that $x\left(s ; \phi_{1}, \lambda_{1}\right)$ is a constant function).

Proof. We suppose the theorem false and try to obtain a contradiction. Thus we can assume $\delta_{0}$ is a compact subset of $X \times \Lambda \times[0, \infty)$, and that if we define $\mathcal{C}$ by

$$
\begin{array}{r}
\mathcal{C}=\{(\phi, \lambda, t) \in X \times \Lambda \times[0, \infty): \phi \text { is a constant function, } \\
f(\phi, \lambda)=0 \text { and } t \geqslant 0\},
\end{array}
$$

then $\delta_{0} \cap \mathcal{C}=\left\{\left(0, \lambda_{0}, t_{0}\right)\right\}$. If $\Lambda=(a, b)$, then the compactness of $\delta_{0}$ implies that there is a positive number $\delta_{1}$ such that $a+\delta_{1} \leqslant \lambda \leqslant b-\delta_{1}$ for all $(\phi, \lambda, t) \in \mathcal{S}_{0}$. Lemma 3 , in conjunction with the assumptions on $\mathcal{S}_{0}$, shows that there exists a positive number $\delta$ such that $t \geqslant 2 \delta$ for all $(\phi, \lambda, t) \in \delta_{0}$.

In the notation of $\S \S 2$ and 3 , we have assumed that $\operatorname{det}\left(\Delta_{\lambda_{0}}(0)\right) \neq 0$. It follows that $\operatorname{det}\left(\Delta_{\lambda}(0)\right) \neq 0$ for $\left|\lambda-\lambda_{0}\right| \leqslant \alpha_{0}\left(\alpha_{0}\right.$ some positive number), and thus $L_{\lambda}(\phi) \neq 0$ for $\phi$ a nonzero constant function and $\left|\lambda-\lambda_{0}\right| \leqslant \alpha_{0}$. This in turn implies that there is a positive number $\mu_{0}$ such that $f(\phi, \lambda) \neq 0$ for $\phi$ a constant function with $0<\|\phi\| \leqslant \mu_{0}$ and $\left|\lambda-\lambda_{0}\right| \leqslant \alpha_{0}$. In particular, there is a bounded, open neighborhood $U_{0}$ in $X \times \Lambda \times[0, \infty)$ of $\left(0, \lambda_{0}, t_{0}\right)$ such that the only elements of $\mathcal{C} \cap \bar{U}_{0}$ are of the form $(0, \lambda, t)$. We can assume also that $t \geqslant \delta$ for $(\phi, \lambda, t) \in U_{0}$. The set $\delta_{0}-U_{0}$ is a compact set containing no elements of the closed set $\mathcal{C}$, so there exists a bounded open neighborhood $\Omega_{1}$ of $\delta_{0}-U_{0}$ such that $\bar{\Omega}_{1} \cap \mathcal{C}$ is empty. Again we can suppose that $t \geqslant \delta$ for $(\phi, \lambda, t) \in \Omega_{1}$. We define $\Omega_{2}=\Omega_{1} \cup U_{0}$, a bounded open neighborhood of $\delta_{0}$.

We now use an argument from [27]. Since $t \geqslant \delta$ for $(\phi, \lambda, t) \in \bar{\Omega}_{2}$, the results of $\S 1$ show that $\delta \cap \bar{\Omega}_{2}=K$ is a compact metric space (we define a norm on $X \times \mathbf{R}^{2}$ by $\left.\|(\phi, \lambda, t)\|^{2}=\|\phi\|^{2}+|\lambda|^{2}+|t|^{2}\right)$. Define $A$ to be $\delta_{0}$, a compact subset of $K$ and write $B=\mathcal{S} \cap \partial \Omega_{2}$; note that $A$ and $B$ are disjoint. It follows from a theorem of Whyburn [28, Chapter 1] that there exist disjoint compact subsets $K_{A}$ and $K_{B}$ of $K$ such that $K=K_{A} \cup K_{B}, A \subset K_{A}$ and $B \subset K_{B}$. If $N_{\varepsilon}\left(K_{A}\right)$ denotes the $\varepsilon$ neighborhood of $K_{A}$, there exists $\varepsilon>0$ such that $\overline{N_{\varepsilon}\left(K_{A}\right)} \cap K_{B}$ is empty and $\overline{N_{\varepsilon}\left(K_{A}\right)} \subset \Omega_{2}$. It follows that if we define $\Omega=N_{\varepsilon}\left(K_{A}\right)$, then $\partial \Omega \cap \mathcal{S}$ is empty; and if $\phi=F(\phi, \lambda, t)$ for $(\phi, \lambda, t) \in \partial \Omega$ then $(\phi, \lambda, t) \in(\partial \Omega) \cap U_{0}$ and $\phi=0$.

Now, following Ize [13], define $d(\phi, \lambda, t)$ to be the distance of $(\phi, \lambda, t)$ to $\bar{\Omega} \cap \mathcal{C}$ and define (for positive $r$ )

$$
H_{r}(\phi, \lambda, t)=\left(\phi-F(\phi, \lambda, t),(d(\phi, \lambda, t))^{2}-r^{2}\right) .
$$

As in [13], we have that $H_{r}(\phi, \lambda, t) \neq 0$ for $(\phi, \lambda, t) \in \partial \Omega$ and $r>0$. For if $\phi=F(\phi, \lambda, t)$ for $(\phi, \lambda, t) \in \partial \Omega$, the remarks above show that $(\phi, \lambda, t) \in \partial \Omega$ 
$\cap U_{0}$ and $\phi=0$. This shows that $d(\phi, \lambda, t)=0$, so that $H_{r}(\phi, \lambda, t)=(0$, $-r^{2}$ ).

If $r$ and $\rho$ are positive numbers, define (for notational convenience)

$$
\begin{aligned}
B(r, \rho)=\{ & (\phi, \lambda, t) \in X \times \Lambda \times[0, \infty): \\
& \left.\|\phi\|^{2}+\left(\lambda-\lambda_{0}\right)^{2}+\left(t-t_{0}\right)^{2} \leqslant r^{2}+\rho^{2}\right\} .
\end{aligned}
$$

It is not hard to show (by using the fact $U_{0} \cap \mathcal{C}=\left\{(0, \lambda, t) \in U_{0}\right\}$ ) that there exist positive numbers $r_{1}$ and $\rho_{1}$ such that $d(\phi, \lambda, t)=\|\phi\|$ for $(\phi, \lambda, t)$ $\in B\left(r_{1}, \rho_{1}\right)$. We can also assume that $T_{\lambda}(t)$ is one-one for $0<\left(\lambda-\lambda_{0}\right)^{2}+(t$ $\left.-t_{0}\right)^{2} \leqslant \rho_{1}^{2}+r_{1}^{2}$. We can suppose that we originally took $U_{0}$ to be $B\left(r_{1}, \rho_{1}\right)$ in our construction of $\Omega_{2}$ and $\Omega$ and that there is a positive number $k$ such that $d(\phi, \lambda, t) \geqslant k$ for $(\phi, \lambda, t) \in \bar{\Omega}-B\left(r_{1}, \rho_{1}\right)$. Select positive numbers $r_{2}<$ $k$ and $\rho_{2} \leqslant \rho_{1}$ such that $B\left(r_{2}, \rho_{2}\right) \subset \Omega$. Finally, take a positive number $r_{3}$ such that

$$
\begin{gathered}
\phi-F(\phi, \lambda, t) \neq 0 \text { for }\|\phi\|=r_{3} \text { and } \\
\rho_{2}^{2} \leqslant\left(\lambda-\lambda_{0}\right)^{2}+\left(t-t_{0}\right)^{2} \leqslant \rho_{1}^{2}+r_{1}^{2} .
\end{gathered}
$$

We claim that $H_{r_{3}}(\phi, \lambda, t) \neq 0$ for $(\phi, \lambda, t) \in \bar{\Omega}-B\left(r_{3}, \rho_{2}\right)$. Since $r_{3} \leqslant k$, it suffices to show that $H_{r_{3}}(\phi, \lambda, t) \neq 0$ for $(\phi, \lambda, t) \in B\left(r_{1}, \rho_{1}\right)-B\left(r_{3}, \rho_{2}\right)$. However, $d(\phi, \lambda, t)=\|\phi\|$ for $(\phi, \lambda, t) \in B\left(r_{1}, \rho_{1}\right)$, so that $\|\phi\|=r_{3}$ if $H_{r_{3}}(\phi, \lambda, t)=0$, and this contradicts (75).

At this point we need to interrupt the proof of Theorem 4 to prove two lemmas.

Lemma 7. Assume $\mathrm{H} 1-\mathrm{H} 5$ and let $\Omega$ be as above. Define $K_{1}=\overline{\mathrm{co}} F(\Omega)$ (where $\overline{c o}$ denotes the closure of the convex hull of a set) and $\Omega_{1}=\{(\phi, \lambda, t) \in \Omega$ : $\left.\phi \in K_{1}\right\}$; generally define $K_{j+1}=\overline{c o} F\left(\Omega_{j}\right)$ and $\Omega_{j+1}=\{(\phi, \lambda, t) \in \Omega: \phi \in$ $\left.K_{j+1}\right\}$. Then the $K_{j}$ and $\Omega_{j}$ form decreasing sequences of sets and there is an integer $N$ such that $K_{j}$ is compact for $j \geqslant N$. If $P$ is a continuous retraction into $K_{N}(P$ exists by results in [5]), the equation

$$
\phi=s F(\phi, \lambda, t)+(1-s) P F(\phi, \lambda, t)
$$

is satisfied for $(\phi, \lambda, t) \in \Omega$ and some $s$ with $0 \leqslant s \leqslant 1$ if and only if

$$
\phi=F(\phi, \lambda, t) .
$$

Proof. The fact that the $K_{j}$ and $\Omega_{j}$ form decreasing sequences of sets is immediate. Since we know that $t \geqslant \delta$ if $(\phi, \lambda, t) \in \Omega$, the results of the first section show that $\{F(\phi, \lambda, t):(\phi, \lambda, t) \in \Omega\}$ is equicontinuous on $[-\delta, 0]$, and it follows immediately that $K_{1}$ is equicontinuous on [- $\left.\delta, 0\right]$. Repeating this argument, we find that $K_{j}$ is equicontinuous on $[-c, 0]$, where $c=$ $\max (-\gamma,-j \delta)$ and $\gamma$ is as in the definition of $X$. It follows that $K_{N}$ is compact if $N \delta \geqslant \gamma$. 
Finally, suppose that (76) holds for some $s$ with $0 \leqslant s \leqslant 1$. Since the right-hand side of (76) is a convex combination of points in $K_{1}$, it follows that $\phi \in K_{1}$ and $(\phi, \lambda, t) \in \Omega_{1}$. Repeating this argument, we find that $\phi \in K_{2}$, $\phi \in K_{3}$ and eventually that $\phi \in K_{N}$. However, if $\phi \in K_{N}$, then $F(\phi, \lambda, t) \in$ $K_{N+1} \subset K_{N}$ and $P F(\phi, \lambda, t)=F(\phi, \lambda, t)$, so that $(\phi, \lambda, t)$ satisfies (77).

The next lemma is crucial for our work. In fact, our main reason for introducing the projections $\left\{P_{n}\right\}$ was to prove the following lemma. We should remark that if $t_{0}>\gamma$, we can prove the lemma without introducing the projections $\left\{P_{n}\right\}$ and with a much simpler proof.

LEMMA 8. Assume $\mathrm{H} 1-\mathrm{H} 5$ and suppose the parity of $i \beta$ is odd. Let $\Omega$ be as above, and let notation be as in Lemma 6 . Then if $P$ is the retraction onto $K_{N}$ defined above, the map

$$
(x, \lambda, t) \in A \rightarrow\left(x-P F(x, \lambda, t),\|x\|^{2}-r^{2}\right) \in(X \times \mathbf{R})-\{0\}
$$

is essential with respect to $B$.

Proof. Consider, for $0<s \leqslant 1$ and $(x, \lambda, t) \in A$, the homotopy

$$
\left(x-(1-s) P_{n} F(x, \lambda, t)-s P F(x, \lambda, t),\|x\|^{2}-r^{2}\right) .
$$

According to Lemma 6 it suffices to show that (78) is nonzero for $n$ large. If not, there will be a subsequence $\left(x_{n_{i}}, \lambda_{n_{i}}, t_{n_{i}}\right) \in A$ and $s_{n_{i}} \in[0,1]$ for which (78) is zero. By taking a further subsequence we can assume (since $P$ is compact) that $P F\left(x_{n_{i}}, \lambda_{n_{n}}, t_{n_{i}}\right) \rightarrow z$. If we define $\Phi(x, \lambda, t, s)=(1-$ $s) F(x, \lambda, t)$ and apply Theorem 1 , we can suppose (by taking a further subsequence) that $\left(x_{n_{i}}, \lambda_{n_{i}}, t_{n_{i}}\right) \rightarrow(x, \lambda, t)$ and $s_{n_{i}} \rightarrow s$. Continuity now implies that

$$
x-(1-s) F(x, \lambda, t)-s P F(x, \lambda, t)=0 \text { and }\|x\|=r .
$$

It follows from Lemma 7 that $\left(x-F(x, \lambda, t),\|x\|^{2}-r^{2}\right)=0$, which is a contradiction.

We can now complete the proof of Theorem 4. Let $r_{3}$ and $\rho_{2}$ be as defined in that proof and suppose also that $0<r_{3}<r\left(\rho_{2}\right)$, where $r(\rho)$ is the function defined in Lemma 4. Modify the definition of $H_{r}, r>0$, so as to make it a compact vector field.

$$
\tilde{H}_{r}(\phi, \lambda, t)=\left(\phi-P F(\phi, \lambda, t),\|\phi\|^{2}-r^{2}\right) .
$$

According to Lemma 7, the zeros of $\tilde{H}_{r}$ and $H_{r}$ are the same, so we know that $\tilde{H}_{r_{2}}(\phi, \lambda, t) \neq 0$ on $\bar{\Omega}-B\left(r_{3}, \rho_{2}\right)$.

The remainder of our proof (now that we have modified $H_{r}$ properly) follows the outlines of [13]. Let $C$ denote a closed ball in $X \times R^{2}$ with center at $\left(0, \lambda_{0}, t_{0}\right)$ and containing $\Omega$. For $r$ large, say $r \geqslant r_{4}$, it is clear that $H_{r} \mid \bar{\Omega}$ is inessential with respect to $C$. Since $H_{r} \mid \partial \Omega$ is nonzero for all $r>0$, it follows 
that $\tilde{H}_{r_{2}} \mid \partial \Omega$ has an extension to a compact vector field $\tilde{\tilde{H}}_{r_{2}}: C \rightarrow(X \times \mathbf{R})$ which is nonzero on $C$. Define $\hat{H}_{r_{2}}: C \rightarrow(X \times \mathbf{R})$ by

$$
\hat{H}_{r_{2}}(\phi, \lambda, t)= \begin{cases}\tilde{H}_{r_{2}}(\phi, \lambda, t) & \text { for }(\phi, \lambda, t) \notin \Omega, \\ \tilde{H}_{r_{2}}(\phi, \lambda, t) & \text { for }(\phi, \lambda, t) \in \Omega .\end{cases}
$$

By our construction we have that

$$
\hat{H}_{r_{2}}(\phi, \lambda, t) \neq 0 \quad \text { for }(\phi, \lambda, t) \notin B\left(r_{2}, \rho_{3}\right)=B \text {. }
$$

Now restrict attention to $A=\Sigma\left(r_{2}, \rho_{3}\right)=\partial B$, and for $(\phi, \lambda, t)$ let $R$ denote the radius of $C$, and define a homtopy $g_{\tau}: A \rightarrow(X \times \mathbf{R})-\{0\}$ through compact vector fields by

$$
g_{\tau}(\phi, \lambda, t)=\hat{H}_{r_{2}}\left(\left(0, \lambda_{0}, t_{0}\right)+\lambda(\tau)\left(\phi, \lambda-\lambda_{0}, t-t_{0}\right)\right)
$$

where $\lambda(\tau)=(1+\tau(R-1))$ and $0 \leqslant \tau \leqslant 1$. By our construction we have that $g_{\tau}(\phi, \lambda, t) \neq 0$ for $(\phi, \lambda, t) \in A$ and $0 \leqslant \tau \leqslant 1$. Since we know that $\hat{H}_{r_{2}} \mid \partial C$ is inessential with respect to $C$, it follows that $g_{1} \mid A$ is inessential with respect to $B$ and, consequently, that $\hat{H}_{r_{2}} \mid A$ is inessential with respect to $B$. However this contradicts Lemma 8 and completes the proof.

REMARK. Of course the difficulty in applying Theorem 4 is the same as for the O.D.E. case: one has little control over the $t$, or period variable, in $(\phi, \lambda, t) \in \mathcal{S}_{0}$. The period need not be the minimal period, and it can happen that $(\phi, \lambda)$ is bounded for $(\phi, \lambda, t) \in \mathcal{S}_{0}$ but $\mathcal{S}_{0}$ is unbounded.

\section{REFERENCES}

1. J. Alexander and J. Yorke, Global bifurcation of periodic orbits (to appear).

2. K. Borsuk, Theory of retracts, Polish Sci. Publ., Warsaw, 1967.

3. Shui-Nee Chow and J. Mallet-Paret, Fuller's index and global Hopf's bifurcation (to appear).

4. K. Cooke and J. Yorke, Equations modelling population growth and gonorrhea epidemiology, Math. Biosci. 16 (1973), 75-101.

5. J. Dugundji, An extension of Tietze's theorem, Pacific J. Math. 1 (1951), 353-367.

6. F. B. Fuller, An index of fixed point type for periodic orbits, Amer. J. Math. 89 (1967), 133-148.

7. R. B. Grafton, A periodicity theorem for autonomous functional differential equations, J. Differential Equations 6 (1969), 87-109.

8. ___ Periodic solutions of certain Lienard equations with delay, J. Differential Equations 11 (1972), 519-527.

9. A. Granas, Theory of compact vector fields, Rozprawy Mat. 30 (1962), 93 pp.

10. J. Hale, Functional differential equations, Springer-Verlag, New York, 1971.

11. E. Hille and R. S. Phillips, Functional analysis and semi-groups, Amer. Math. Soc. Colloq. Publ., vol. 31, rev. ed., Amer. Math. Soc., Providence, R.I., 1957.

12. J. Ize, Bifurcation theory for Fredholm operators, Mem. Amer. Math. Soc., no. 174, 1976.

13. , Global bifurcation of periodic orbits, Communicaciones Tecnicas of C.I.M.A.S., Vol. 5, Series B., No. 8 (1974). (Spanish)

14. G. S. Jones, The existence of periodic solutions of $f^{\prime}(x)=-\alpha f(x-1)[1+f(x)]$, J. Math. Anal. Appl. 4 (1962), 440-469. 
15. Periodic motions in Banach space and applications to functional differential equations, Contrib. Differential Equations 3 (1964), 75-106.

16. J. Kaplan and J. Yorke, Ordinary differential equations which yield periodic solutions of differential-delay equations, J. Math. Anal. Appl. 48 (1974), 317-324.

17. T. Kato, Perturbation theory for linear operators, Springer-Verlag, New York, 1966.

18. J. Mallet-Paret, Generic periodic solutions of functional differential equations (to appear).

19. R. May, Stability and complexity in model ecosystems, Princeton Univ. Press, Princeton, N.J., 1973.

20. L. Nirenberg, Topics in nonlinear functional analysis, New York Univ. Lecture Notes, 1973-1974.

21. R. D. Nussbaum, A global bifurcation theorem with applications to functional differential equations, J. Functional Analysis 19 (1975), 319-338.

22. __ Pericdic solutions of some nonlinear autonomous functional differential equations. II, J. Differential Equations 14 (1973), 360-394.

23. , Global bifurcation of periodic solutions of some autonomous functional differential equations, J. Math. Anal. Appl. 55 (1976), 699-725.

24. Appl. 58 (1977), 280-292.

25. __ Periodic solutions of differential-delay equations with two time lags (submitted).

26. W. V. Petryshyn, On the approximation-solvability of equations involving A-proper and pseudo-A-proper mappings, Bull. Amer. Math. Soc. 81 (1975), 223-312.

27. P. Rabinowitz, Some global results for nonlinear eigenvalue problems, J. Functional Analysis 7 (1971), 487-513.

28. G. T. Whyburn, Topological analysis, Princeton Univ. Press, Princeton, N.J., 1958.

Department of Mathematics, Rutgers University, New Brunswick, NeW Jersey 08903 